\title{
Vegetation of the Okavango River valley in Kavango West, Namibia
}

\author{
Ben J. Strohbach
}

\begin{abstract}
The vegetation of the Okavango River Valley has previously only been described in general terms along the gradient from base of the valley to the top of the sand plateau, without the clear definition of different vegetation types or distinct vegetation composition. Yet the valley bottom supports a large part of the population within the region, who are dependent on ecosystem services provided by the vegetation. A Braun-Blanquet type survey was conducted to describe the vegetation of the Okavango River Valley in the Kavango West Region of Namibia, with special emphasis on the floodplain vegetation. The survey followed the guidelines set out for the "Vegetation Survey of Namibia" project. The data was classified using the modified TWINSPAN procedure in Juice, resulting in four higher-order syntaxa and 11 vegetation associations. We described these associations informally according to diagnostic species, species richness and environmental variables. In the case of floodplain and wetland associations, information regarding the flooding regime (depth, duration and probability of flooding) is provided. Although the higher-order groupings within this association come out very clearly, only the wetlands could be related to similar higher-order syntaxa described for the oshana wetlands in the Cuvelai delta. The placement and validity of the other three higher-order syntaxa is unclear at this stage, and needs to be addressed in a synopsis of all existing vegetation descriptions for the greater Kalahari ecoregion. The various vegetation associations can very clearly be associated with distinct positions in the landscape. The degree of flooding at the one extreme, and the sandiness of the soil on the other extreme, defines the vegetation type and composition. The Acacia erioloba-Schinziophyton rautanenii association (7) has been cleared and ploughed to at least $90 \%$, with only small patches of this vegetation remaining. Expansion of fields into the sands of the terrace embankment is worrisome, leading to fears of further soil degradation and ultimately desertification. Although signs of overgrazing are visible within the floodplains, this cannot be distinctly quantified as yet. Overgrazing in the wetlands will lead to the destruction of fish spawning and breeding habitats, damaging the other major food source of the people of the region.
\end{abstract}

Keywords: Braun-Blanquet; desertification; floodplain vegetation; flooding regime; land degradation; land use; phytosociology; seasonal wetland vegetation.

\section{A vegetação do vale do Rio Okavango no Kavango West, Namíbia}

Resumo: A vegetação do vale do Rio Okavango foi apenas descrita previamente em termos gerais ao longo da encosta, da base do vale até o topo do platô de areia, sem uma definição clara dos diferentes tipos de vegetação ou sua composição distinta. No entanto, o fundo do vale auxilia uma grande parte da população da região, que é dependente dos serviços ecosistemicos fornecidos pela vegetação. Uma pesquisa do tipo "Braun- Blanquet" foi feita para descrever a vegetação do vale do Rio Okavango, na região oeste do Kavango na Namíbia, com ênfase especial na vegetação de várzea. O estudo seguiu as diretrizes estabelecidas para o projeto "Estudo da Vegetação da Namíbia". Os dados foram classificados usando o procedimento modificado TWINSPAN em Juice, resultando em quatro syntaxa de ordem superior e 11 associações de vegetação. Descrevemos essas associações informalmente de acordo com diagnóstico de espécies, riqueza das espécies e variações ambientais. No caso de associações de várzea e pantano, as informações sobre o regime de inundação (profundidade, duração e probabilidade de inundação) são fornecidas. Embora os grupos de ordem superior dentro dessa associação apareçam muito claramente, apenas os pantanos podem estar relacionados com syntaxa de ordem superior similar descrita nos pantanos Oshana, no delta Cuvelai. A colocação e validade dos outros três syntaxa de ordem superior não estão claras nesta fase e precisam ser tratadas em uma sinopse de todas as descrições de vegetação existentes, para a vasta ecoregião de Kalahari. As diversas associações de vegetação podem ser claramente associadas com posições distintas na paisagem. O grau de inundação em um extremo e a arenosidade do solo no outro extremo, definem o tipo e a composição da vegetação . A associação Acacia erioloba -Schinziophyton rautanenii (7) foi removida e arada em pelo menos 90\%, remanescendo apenas pequenos fragmentos dessa vegetação. A expansão dos campos nas areias da plataforma do aterro é preocupante, levando a temores de maior degradação do solo e, finalmente, a desertificação. Apesar de sinais de sobrepastoreio serem vísiveis dentro das várzeas, isto ainda não pode ser quantificado distintamente. O sobrepastoreio nos pantanos levará à destruição da desova e habitats de reprodução dos peixes, prejudicando a outra grande fonte de alimento dos povos da região..

Palavras-chave: Braun-Blanquet; degradação do solo; desertificação; fitossociologia; regime de inundação; uso do solo; vegetação sazonal de pantanos; vegetação de várzea.

Received: 08 November 2013 - Accepted: 19 December 2013 


\section{Introduction}

The Okavango River, having it's headwaters in central Angola, flows in a generally south-westerly direction, ending in a large swamp area in the form of the Okavango Delta in northern Botswana (Mendelsohn \& el Obeid 2004). As part of it's course, it forms, for roughly $415 \mathrm{~km}$, the border between Angola and Namibia before crossing Namibia and entering Botswana. The river forms a deeply incised V-shaped valley in an otherwise flat landscape formed by the Kalahari sand plateau. The altitude of the river ranges between $1100 \mathrm{~m}$ upon entering Namibia along the Angolan border and $1000 \mathrm{~m}$ when leaving Namibia into Botswana. The river is roughly 40 to $60 \mathrm{~m}$ lower than the surrounding sand plateau (Mendelsohn \& el Obeid 2003; own observations).

The river is estimated to be ca 65 Million years old, and has likely flowed for considerable time at a far higher volume than at present (Mendelsohn \& el Obeid 2004). Alluvial deposits from this high flow regime formed the relatively fertile valley bottom, compared to the nutrientpoor sands of the surrounding Kalahari sand plateau (Mendelsohn \& el Obeid 2004, Schneider 1986). During the relatively low-flow period over the past 2 million years, the actual stream bed started to meander through the valley bottom, forming an extensive floodplain, often with temporary marshes and oxbow lakes (Mendelsohn \& el Obeid 2004). Three distinct regions of extensive floodplains can be distinguished along the Namibian section of the Okavango River: The western floodplains, reaching from west of Tondoro (towards Nkurenkuru) to Shambyu (east of Rundu) and the floodplains at the confluence of the Cuito and the Okavango are both of strongly seasonal character. The eastern floodplains below the Popa Falls are of a more permanent flooded nature, forming the beginning of the "Panhandle" of the Okavango Delta (Hay et al. 1996, Mendelsohn $\&$ el Obeid 2003). The Okavango flows at its peak during April each year, with high variability in water levels between peak and low season (Fig. 1). The Cuito brings less water during peak flow periods (April - May) than the Okavango, but is also far less variable in flow throughout the seasons (Bethune 1991).

The climate of the region can best be described as a transition between a subtropical steppe and subtropical (sub)humid climate (sensu Köppen 1936) (following data provided in Mendelsohn et al. 2002). The average annual rainfall at Rundu is $445 \mathrm{~mm}$, with the peak rainfall months being January and February each year (Namibia Meteorological Services 1997) (Fig. 2). The co-efficient of variation for the rainfall is 36\% (Botha 1996).

Human settlement started some 1,500 to 1,000 years ago, when the Kwangali, Mbunza, Shambyu and Gciriku tribes migrated from central and eastern Africa into this area. Population densities remained relatively low, with a population of less than 50000 people in the Okavango district in the 1970's (Mendelsohn \& el Obeid 2004). The present-day Kavango East and West regions in Namibia support a population of 222,500 people, of which 61,900 are residing in the town of Rundu (National Planning Commission 2012). The remainder of the mostly rural population mainly lives in the valley along the Kavango River, with population densities of between 10 to 40 people per $\mathrm{km}^{2}$ (Mendelsohn \& el Obeid 2003). There is a striking difference in population density between the Namibian and Angolan side of the Okavango valley, with the Angolan side being far less populated than the Namibian side due to the war (Mendelsohn \& el Obeid 2003). The Kavango has the highest average household size in the country (6.0 persons per household), and in general has some of the poorest people in the country (Central Bureau of Statistics 2011, National Planning Commission 2012). The general livelihood of the people is derived from small-scale agropastoralism, supported by fishing (Mendelsohn \& el Obeid 2003, Mendelsohn et al. 2006). There is thus an overall strong dependency on the natural environment for survival by the people. This led, over years, to severe deforestation and land degradation along the Okavango River valley, with over $95 \%$ of the original vegetation estimated to have been destroyed. The expanding Green Scheme contributes to this threat (Hofmeyr 2004).

Although extensive, in-depth studies have been done on the permanent wet-

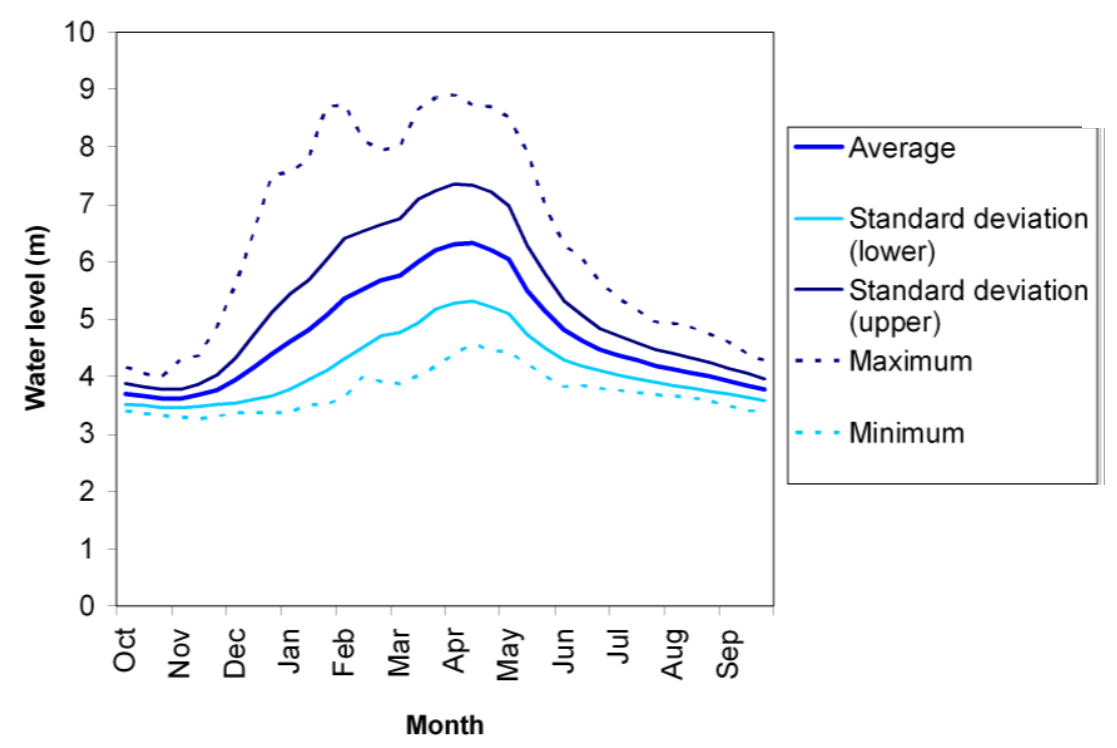

Fig. 1: Water levels of the Okavango River at Rundu gauging station between 1945 2012. Data source: Okavango Basin Information System (http://leutra.geogr.unijena.de/obis/metadata/start.php) and Directorate Hydrology, Ministry of Agriculture, Water and Forestry.

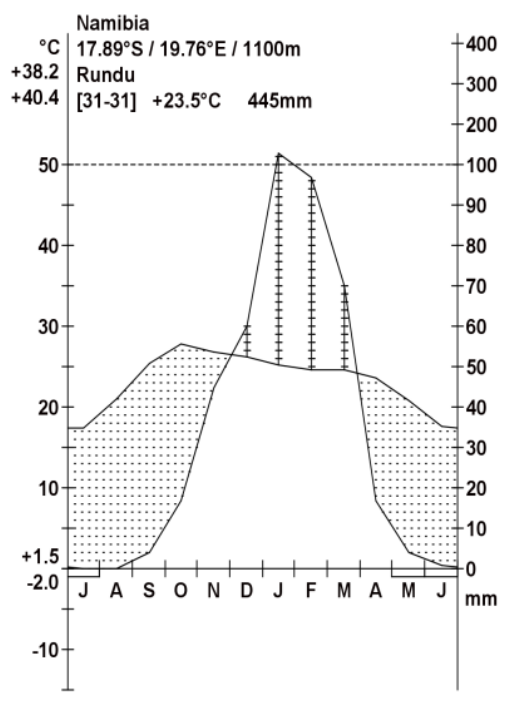

Fig. 2: Climate diagram for Rundu. Data source: Okavango Basin Information System (http://leutra.geogr.uni-jena.de/ obis/metadata/start.php) and Namibia Meteorological Services (1997). 
lands of the Okavango River Delta in Botswana (Biggs 1976, Bonyongo 1999, Ellery et al. 1991, Gumbricht et al. 2004, Tooth \& McCarthy 2004), little is known about the vegetation of the Okavango River Valley within the middle section of the river, especially the vegetation beyond the wetland system associated with the river. The eastern and western Kavango regions fall within the broader "Dry Forests and Woodlands of the northern Kalahari" sensu Giess (1998), or the "Zambesian Baikiaea Woodlands" (Vetter 2001). The Okavango valley vegetation responds to the variation in soil types along the catena from river bed to sand plateau (Burke 2002, De Sousa Correia \& Bredenkamp 1986). This is schematically illustrated by Mendelsohn and el Obeid (2003). Bethune (1991) describes the wetlands of the Kavango river, without giving detail of spatial distribution of these wetlands. She distinguishes between "open water, river margins, reed fringes, riparian forests and thickets, floodplains, permanent marshes and alluvial terraces". For these landscape elements, dominant plant species are listed, but no phytosociological definition or description is offered. The aim of this study is thus to define and describe the various vegetation types of the Okavango Valley within the western Kavango region.

\section{METHODS}

\section{Field survey}

A Braun-Blanquet type survey of the vegetation was undertaken during February 2012, and again in February and March 2013. Relevés were sampled on 20x50 m plots, in line with the standard set for the Namibian Vegetation Survey (Strohbach 2001). This also corresponds to the suggested plot sizes for savanna vegetation (Brown et al. 2013). Plots were set out systematically along paths and cutlines from the main road to the Okavango River, as well as towards the hinterland. These were concentrated in the area between Tondoro and Rundu, with some outliers in the Mashare area (range: $17^{\circ} 43^{\prime} 28^{\prime}$ 'S 18 $44^{\prime} 02^{\prime \prime}$ E up to $17^{\circ} 53^{\prime} 05^{\prime}$ 'S $20^{\circ} 12^{\prime} 36^{\prime \prime E}$ ). At each plot, the location was determined with a Garmin e-Trex GPS. In addition, habitat descriptors relating to the landscape and local topography, slope, stoniness (if any), parent material, severity of erosion and type of disturbances were recorded. At least one photo was taken at each plot. At

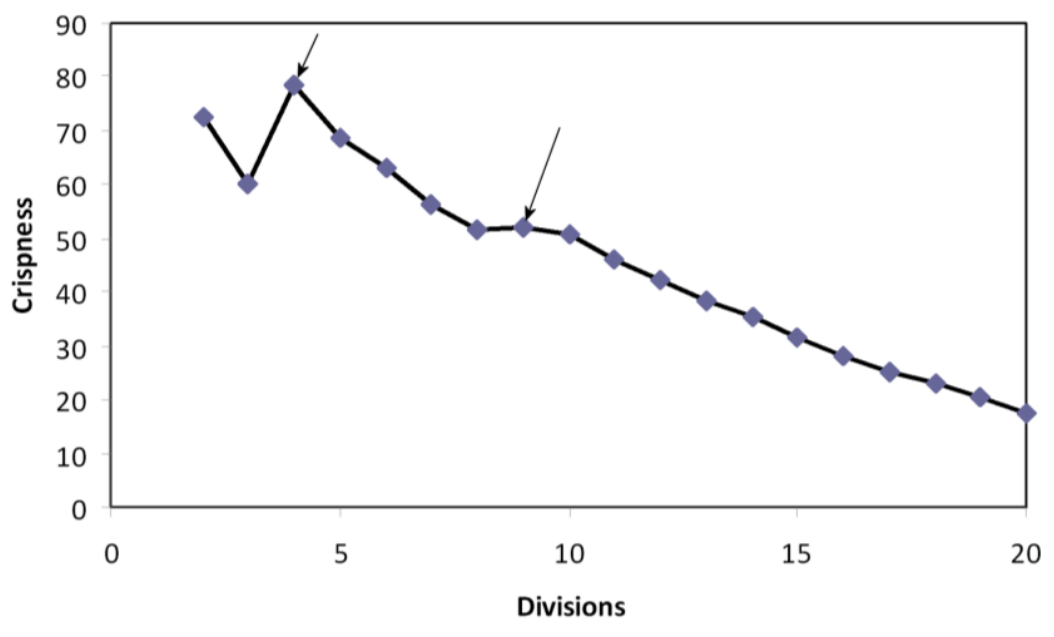

Fig. 3: Crispness scores as calculated for the modified TWINSPAN classification of relevés. Peaks for significant levels of divisions (Botta-Dukát et al. 2005) are indicated by arrows (at 4 and 9 divisions) in the graph.

several representative plots, soil samples by way of augering were taken of the topsoil and if possible, of the lower horizon. All vascular plant species were recorded, together with the average height and growth form of the plants, and an estimated crown cover percentage (Strohbach 2012). Unknown plant species were sampled, pressed and identified at the National Herbarium of Namibia (WIND). In the case of wetland vegetation, the survey was conducted by wading into the water or by using a canoe.

\section{Data Processing}

The vegetation data was captured in TurboVeg (Hennekens \& Schaminée 2001) and exported to Juice (Tichý 2002). In order to avoid observer bias, species which were likely to be confused during field surveys (e.g. Acrotome inflata and A. angustifolia), or in some cases different subspecies of a species (e.g. three subspecies of Acacia hebeclada), were combined, before the relevés were classified with the modified TWINSPAN procedure (Roleček et al. 2009). As distance measure, average Jaccard was used, together with three pseudospecies cut levels set at $0 \%, 5 \%$ and $20 \%$ respectively. Crispness scores (Botta-Dukát et al. 2005) were used to determine the optimal number of divisions. Further refinements were done to the classification, involving the splitting of Cluster 1 into three separate clusters using Cocktail procedures (Bruelheide 2000).

\section{Flooding levels}

A number of identified vegetation units are wetland units, and many others are floodplain vegetation not regularly inundated. The flooding regime (frequency, duration and depth of inundation) is thus an important habitat variable (Biggs 1976, Ellery et al. 1991, Gumbricht et al. 2004). These parameters were calculated for each plot as follows: Daily water level data for the period 1945 to 2012 of the Okavango River at Rundu (source: Okavango Basin Information System: http://leutra.geogr.uni-jena.de/obis/ metadata/start.php and Directorate Hydrology, Ministry of Agriculture, Water and Forestry) was averaged into decadal (10-daily) data. The average water depth for these 36 decades per year was calculated, as well as the minimum, maximum and standard deviations. These figures were graphed to give a visual interpretation of water levels of the Okavango River, especially to determine the duration of the flowing season (from minimum to minimum flow, or from decade 28 - early October - to decade 27 - end September) (Fig. 1). For the same decadal data, the $10^{\text {th }}, 20^{\text {th }}, 30^{\text {th }}, 40^{\text {th }}, 50^{\text {th }}, 60^{\text {th }}$, $70^{\text {th }}, 80^{\text {th }}$ and $90^{\text {th }}$ percentiles were worked out. Together with minimum and maximum, these were interpreted as probabilities of flooding depth in $10 \%$ increments (minimum: $100 \%, 10^{\text {th }}$ percentile: $90 \%$, etc.).

The height of the plots above the nearest point of the river was determined using the elevation profile function of Google Earth. These relative heights above water level were averaged for each 


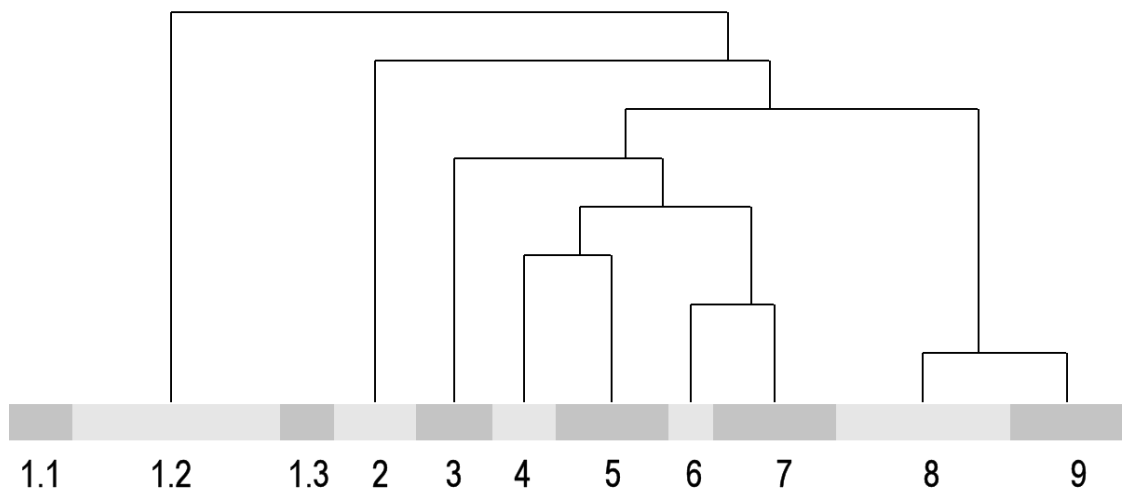

Fig. 4: Dendrogram of the modified TWINSPAN classification of relevés. Cluster 1 was further subdivided into three associations using Cocktail procedures.

vegetation unit, and compared to the table of water depth percentiles for each decade in order to determine the depth, duration and flooding probability of each vegetation unit.

\section{Environmental gradients}

The soil samples were analyzed by the Agricultural Laboratory of the Ministry of Agriculture, Water and Forestry for the following: percentages sand, silt and clay, $\mathrm{pH}$ in water, electric conductivity, cation exchange capacity, phosphorous, sodium, calcium, magnesium, potassium and organic carbon. As only representative soil samples were taken, these values were averaged for each vegetation unit. Together with the flooding regime data, these were used as environmental varia-
These belong to 76 families, of which the Poaceae (90 species), Fabaceae (60 species), Asteraceae (18) and Cyperaceae (18) are the most abundant. 56 Aquatic species were observed on the relevés, as well as 92 phanerophytic (tree and shrub) species.

Crispness values (Botta-Dukát et al. 2005) indicated an ideal classification with either four or nine divisions (Fig. 3). The top four divisions are interpreted as being equivalent to vegetation classes, whilst the final nine divisions (with the exception of Cluster 1) are interpreted as associations. A dendrogram of the classification is depicted in Figure 4.

Cluster 1 was divided into three subclusters, based a) on the combined presence of Mimosa pigra, Searsia quartiniana, Phragmites australis, Mikania sagittifera and Syzygium guineense (seven relevés) and b) the presence of Tacazzea apiculata, as well as by the high abundance of Chrysopogon nigritanus (six relevés). In most cases the fidelity of these species improved after subdivision, whilst the average positive fidelity and sharpness of classification remained fairly high, indicating that such a subdivision is appropriate. Due to the difference in habitat, these subclusters are recognised as associations (Table 1).

A shortened version of the synoptic table is presented in Table 2 below, whilst the full phytosociological table is presented as a downloadable online appendix. The identified associations are described in full in the following section.

Table 1: Refinements done with Cocktail to the modified TWINSPAN-based classification. F: Average positive fidelity, S: sharpness.

\begin{tabular}{|c|c|c|c|c|c|c|}
\hline & \multicolumn{2}{|c|}{ Cluster } & \multicolumn{4}{|c|}{ Fidelity } \\
\hline & Original & Revised & Diagnostic species & Original & Revised & No of relevés involved \\
\hline \multirow[t]{6}{*}{1.1} & F31 & F22 & Mimosa pigra & 40 & 92 & 7 split off \\
\hline & S86 & S51 & Phragmites australis & 64 & 80 & \\
\hline & & & Searsia quartiniana & 20 & 85 & \\
\hline & & & Mikania sagittifera & 28 & 64 & \\
\hline & & & Cyperus digitatius & 28 & 64 & \\
\hline & & & Syzygium guineense & 23 & 52 & \\
\hline \multirow[t]{7}{*}{1.2} & & F28 & Nymphaea nouchali & - & 55 & 22 remaining \\
\hline & & S46 & Paratheria prostrata & 66 & 52 & \\
\hline & & & Oryza longistaminata & 60 & 53 & \\
\hline & & & Schoenoplectus corymbosus & 72 & 43 & \\
\hline & & & Eleocharis acutangula & 43 & 55 & \\
\hline & & & Utricularia stellaris & 40 & 50 & \\
\hline & & & Nymphaea lotus & 49 & 48 & \\
\hline \multirow[t]{3}{*}{1.3} & & $\mathrm{~F} 27$ & Tacazzea apiculata & 37 & 77 & 6 split off \\
\hline & & S33 & Chrysopogon nigritanus & 24 & 40 & \\
\hline & & & Heliotropium baclei & 15 & 61 & \\
\hline
\end{tabular}




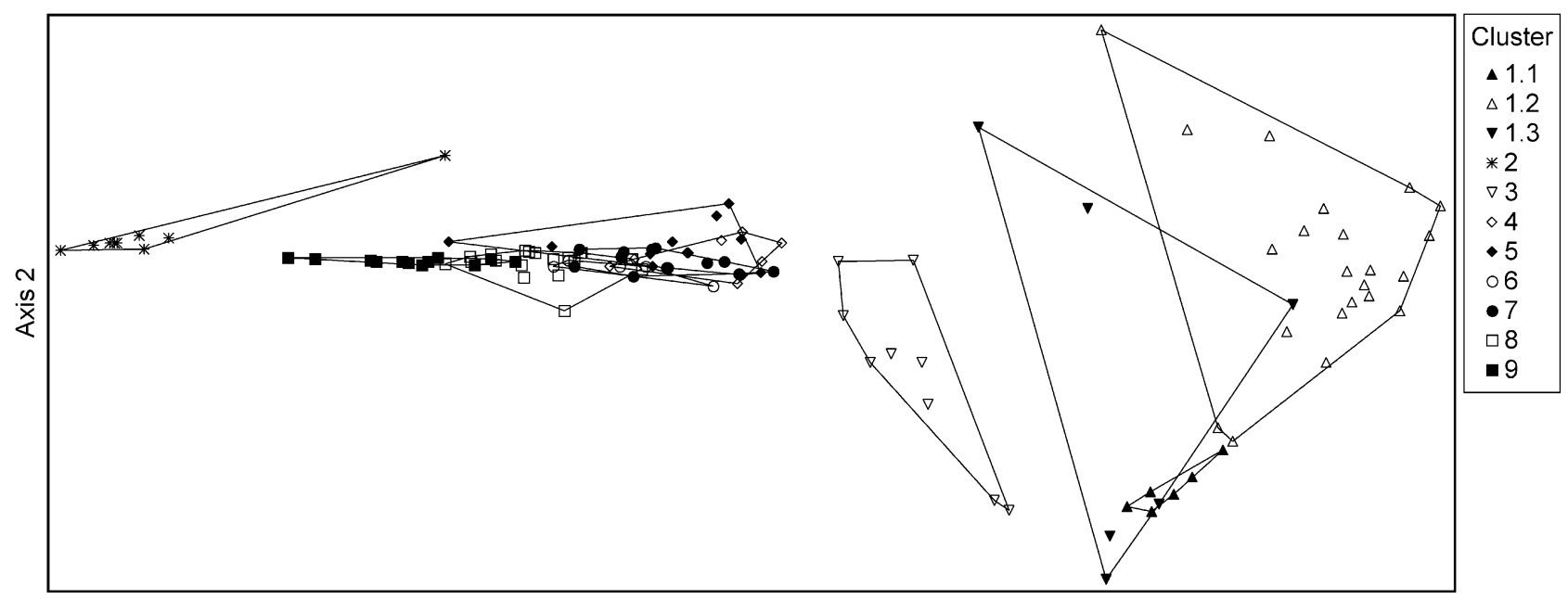

Axis 1

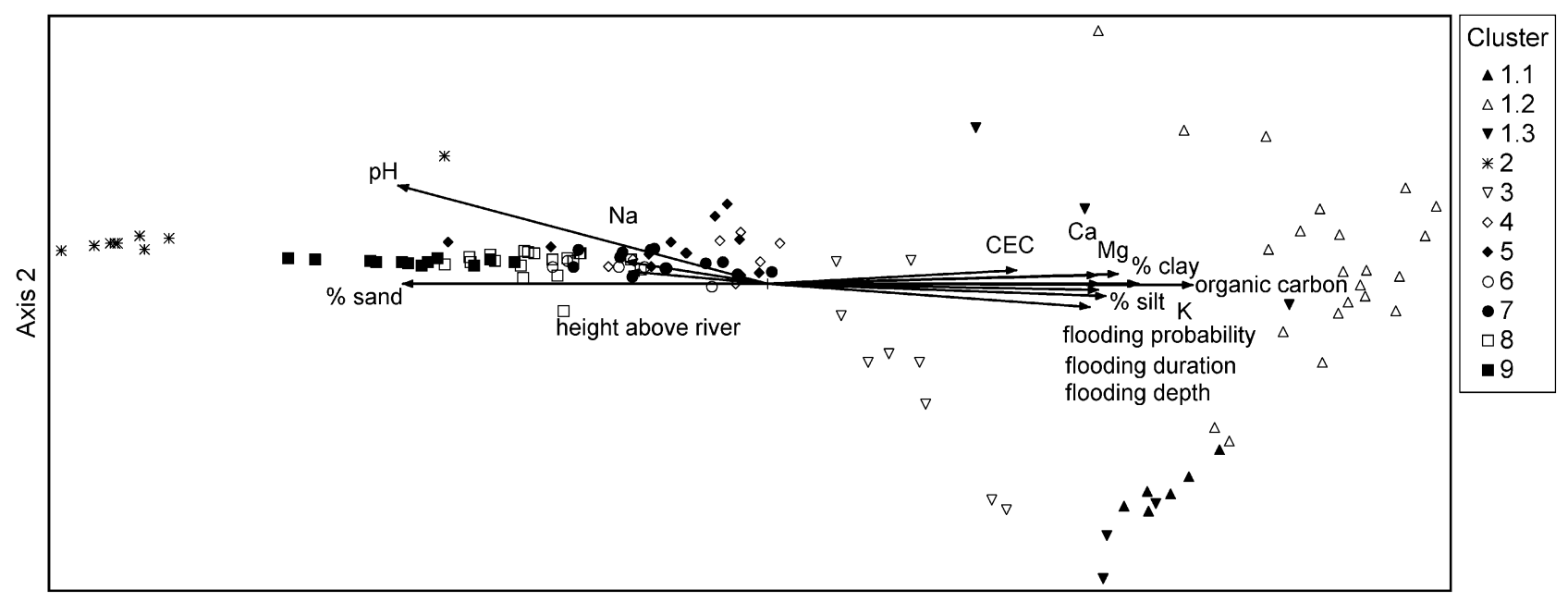

Axis 1

Fig. 5: Top: DCA ordination scatter plot, indicating the various clusters. Bottom: DCA ordination scatter plot with the habitat factors superimposed.

The influence of the habitat is illustrated in Figure 5 by way of an ordination diagram with the known environmental factors superimposed as biplot. Whereas the wetland vegetation is dependent on the flooding regime and occurring on relatively loamy, even clayey soils, the remainder of the valley vegetation forms an elongated gradient away from regular flooding and with ever increasing sandiness of the soils. Cluster 2, the Hakusembe grasslands, form an outlier on fairly sandy, but saline soils with an extremely high $\mathrm{pH}$. The averaged soil characteristics for the various associations, as used in the ordination, are presented in Table 3.

\section{Vegetation associations of the western Okavango river valley}

From the classification results, 11 vegetation associations were identified. Their description follows below. No attempt was made to formally describe these associations following the rules of the International Code for Phytosociological Nomenclature (Weber et al. 2000), as a number of these association descriptions are still based on a very limited number of relevés, whilst some clusters displayed a high internal variation (likely due to the degraded nature of the vegetation), that no clear conclusions can be made at this stage to the syntaxonomic status of it. The numbering of the associations follows that of the initial classification as displayed in Figure 4, and as used in the remainder of the results. An analysis of the species richness of these associations is presented in Figure 8.

\subsection{Mimosa pigra-Phragmites australis association of the river banks}

This association is characterized by the diagnostic species Mimosa pigra, Searsia quartiniana, Phragmites australis, Mikania sagittifera, Cyperus digitatus subsp. auricomus, Aeschynomene fluitans, Syzygium guineense, Persicaria senegalensis, Nymphaea nouchali var. caerulea as well as the constant occurrence of Gardenia volkensii subsp. spatulifolia, Chrysopogon nigritanus, Schoenoplectus corymbosus, Oryzidium barnardii. Seven relevés have been classified into this 
Table 2: Shortened synoptic table of the associations identified within the study area. Only diagnostic species with a fidelity phi coefficient of above $\mathbf{4 0}$ are displayed. On the left, the phi coefficient of the fidelity is presented; on the right the percentage frequency of occurrence of these species is presented.

\begin{tabular}{|c|c|c|c|c|c|c|c|c|c|c|c|c|c|c|c|c|c|c|c|c|}
\hline \multirow[b]{2}{*}{ Association } & \multirow{2}{*}{\multicolumn{11}{|c|}{ phi coefficient }} & \multicolumn{9}{|c|}{ percentage frequency } \\
\hline & & & & & & & & & & & & 1.1 & $\begin{array}{l}1 \\
2\end{array}$ & $\begin{array}{l}1 . \\
3\end{array}$ & 2 & 3 & 4 & 5 & 6 & \\
\hline $\begin{array}{l}\text { No of relevés } \\
\text { Mean no of }\end{array}$ & 7 & 22 & 6 & 9 & 8 & 7 & 12 & 5 & 13 & 19 & 12 & 7 & 2 & 6 & 9 & 8 & 7 & 12 & 5 & \\
\hline $\begin{array}{l}\text { species } \\
\text { No of diagnostic }\end{array}$ & 11 & 10 & 11 & 7 & 14 & 26 & 24 & 35 & 28 & 38 & 44 & 11 & 10 & 11 & 7 & 14 & 26 & 24 & 3 & \\
\hline species & 9 & 10 & 5 & 8 & 5 & 9 & 9 & 37 & 4 & 7 & 39 & 9 & 10 & 5 & 8 & 5 & 9 & 9 & 3 & \\
\hline
\end{tabular}

Diagnostic species

of single groups

Association 1.1: Mimosa pigra-Phragmites australis association

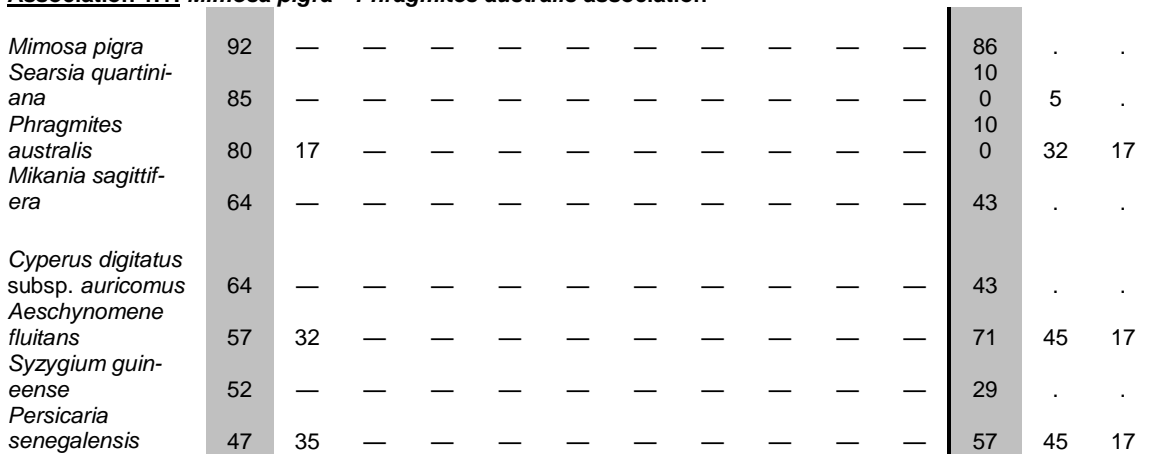

\begin{tabular}{|c|c|c|c|c|c|c|c|c|c|c|c|c|c|}
\hline Eleocharis & & & & & & & & & & & & & \\
\hline $\begin{array}{l}\text { acutangula } \\
\text { Oryza }\end{array}$ & - & 55 & - & - & - & - & - & - & - & - & - & . & 32 \\
\hline $\begin{array}{l}\text { longistaminata } \\
\text { Utricularia }\end{array}$ & - & 53 & - & - & - & - & - & - & - & - & - & 29 & 50 \\
\hline stellaris & - & 50 & - & - & - & - & - & - & - & - & - & . & 27 \\
\hline $\begin{array}{l}\text { Nymphaea lotus } \\
\text { Paratheria }\end{array}$ & - & 48 & - & - & - & - & - & - & - & - & - & 14 & 36 \\
\hline $\begin{array}{l}\text { prostrata } \\
\text { Ipomoea }\end{array}$ & - & 45 & 40 & - & - & - & - & - & - & - & - & 14 & 55 \\
\hline $\begin{array}{l}\text { aquatica } \\
\text { Panicum colora- }\end{array}$ & - & 44 & - & - & - & - & - & - & - & - & - & 14 & 32 \\
\hline tum & - & 42 & - & - & - & - & - & - & - & - & - & 14 & 45 \\
\hline
\end{tabular}

Association 1.3: Tacazzea apiculata-Chrysopogon nigritanus association

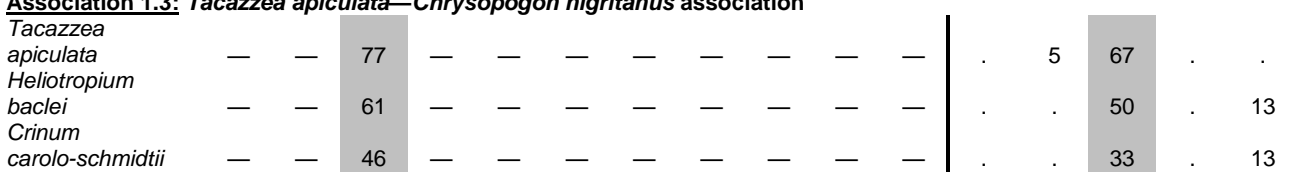

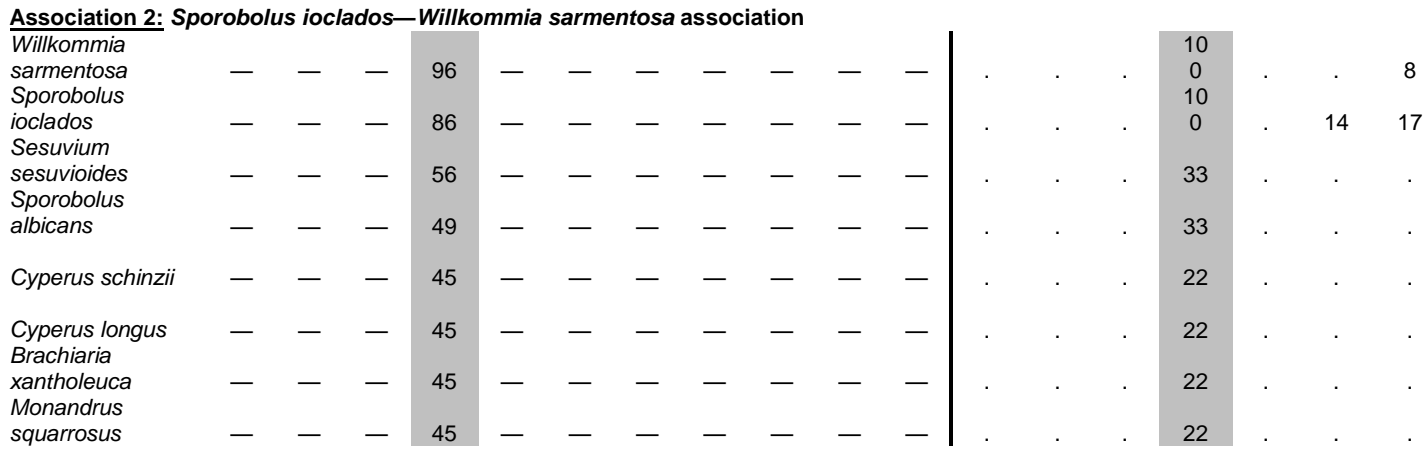

Association 3: Chrysopogon nigritanus-Gardenia volkensii association

$$
\text { Geigeria }
$$$$
\text { nianganensis }
$$

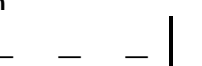

$5 \quad 17$ 
Aristida junci-

formis subsp.

junciformis

Eragrostis

annulata

Nesaea rigidula

Brachiaria

humidicola

$$
\begin{array}{ccccccccccc}
- & - & - & - & 49 & - & - & - & - & - & - \\
- & - & - & - & 48 & - & - & - & - & - & - \\
- & - & - & - & 48 & - & - & - & - & - & - \\
- & - & - & - & 46 & - & - & - & - & - & -
\end{array}
$$

Association 4: Terminalia sericea-Combretum imberbe association

\begin{tabular}{|c|c|c|c|c|c|c|c|c|c|c|c|}
\hline $\begin{array}{l}\text { Euclea undulata } \\
\text { Andropogon }\end{array}$ & - & - & - & - & - & 61 & - & - & - & - & - \\
\hline $\begin{array}{l}\text { schirensis } \\
\text { Asparagus }\end{array}$ & - & 一 & - & - & - & 52 & - & - & - & - & - \\
\hline racemosus & - & 一 & 一 & - & - & 52 & - & - & - & 一 & - \\
\hline Acacia hebeclada & - & 一 & - & - & - & 50 & - & - & - & 一 & - \\
\hline $\begin{array}{l}\text { Kyllinga alba } \\
\text { Pogonarthria }\end{array}$ & - & 一 & 一 & - & - & 49 & - & - & - & 一 & - \\
\hline squarrosa & - & 一 & - & - & - & 46 & - & - & - & 一 & 23 \\
\hline $\begin{array}{l}\text { Ehretia rigida } \\
\text { Combretum }\end{array}$ & - & - & 一 & - & - & 44 & - & - & - & - & - \\
\hline imberbe & - & 一 & - & - & - & 41 & 20 & 28 & - & 一 & - \\
\hline Acacia luederitzii & - & - & - & - & - & 40 & - & - & - & - & - \\
\hline
\end{tabular}

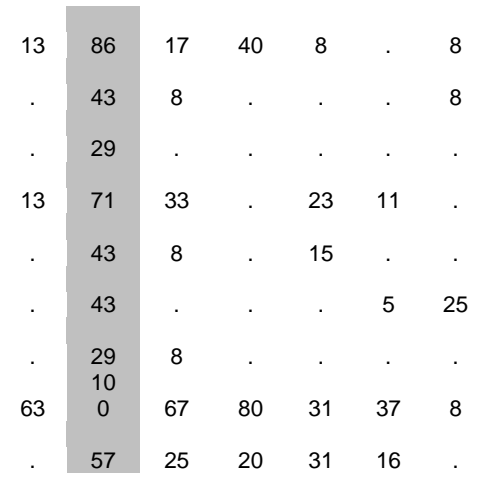

Association 5: Acacia tortilis-Hyphaene petersiana association

\begin{tabular}{|c|c|c|c|c|c|c|c|c|c|c|c|}
\hline $\begin{array}{l}\text { Acacia tortilis } \\
\text { Ipomoea }\end{array}$ & - & - & - & - & - & - & 58 & - & - & - & - \\
\hline adenioides & - & - & - & - & - & - & 56 & - & - & - & - \\
\hline $\begin{array}{l}\text { Chloris virgata } \\
\text { Eragrostis }\end{array}$ & - & - & - & - & - & - & 53 & - & - & - & - \\
\hline $\begin{array}{l}\text { trichophora } \\
\text { Pergularia }\end{array}$ & - & - & - & - & 31 & 29 & 49 & - & 17 & - & - \\
\hline $\begin{array}{l}\text { daemia } \\
\text { Sansevieria }\end{array}$ & - & - & - & - & - & - & 48 & - & - & - & - \\
\hline $\begin{array}{l}\text { aethiopica } \\
\text { Indigofera }\end{array}$ & - & - & - & - & - & - & 48 & - & - & - & - \\
\hline $\begin{array}{l}\text { rautanenii } \\
\text { Urochloa }\end{array}$ & - & - & 一 & - & - & - & 43 & - & 23 & - & - \\
\hline oligotricha & - & - & - & - & - & - & 40 & - & - & - & - \\
\hline
\end{tabular}

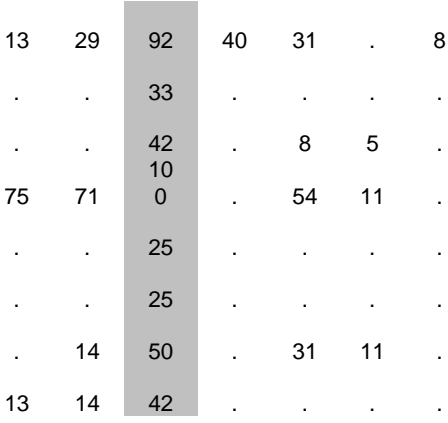

\section{Association 6: Acacia sieberiana-Ficus association}

$$
\begin{aligned}
& \text { Ocimum }
\end{aligned}
$$

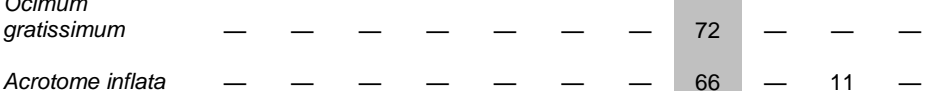

$$
\begin{aligned}
& \text { Senna } \\
& \text { occidentalis } \\
& \text { Jasminum } \\
& \text { Jasminum } \\
& -\quad-\quad-\quad-\quad-\quad-\quad-61 \quad-6-6 \\
& \text { Hibiscus caesius } \\
& \text { Abutilon } \\
& \text { angulatum } \\
& \text { Senna obtusifolia } \\
& \begin{array}{l}
\text { Setaria verticillata } \\
\text { Panicum }
\end{array} \\
& \text { maximum } \\
& \text { marlothii } \\
& \text { divaricatum } \\
& \text { Hermannia } \\
& \text { guerkeana } \\
& \text { integrifolius } \\
& \text { Asparagus nelsii } \\
& -\quad-\quad-\quad- \\
& -\quad- \\
& \begin{array}{r}
59 \\
59
\end{array} \\
& -\quad- \\
& -\quad- \\
& -\quad-\quad- \\
& \text { - }-56
\end{aligned}
$$$$
533
$$$$
\begin{array}{l|l}
38 & 14 \\
25 & . \\
25 & . \\
63 & 29
\end{array}
$$ 


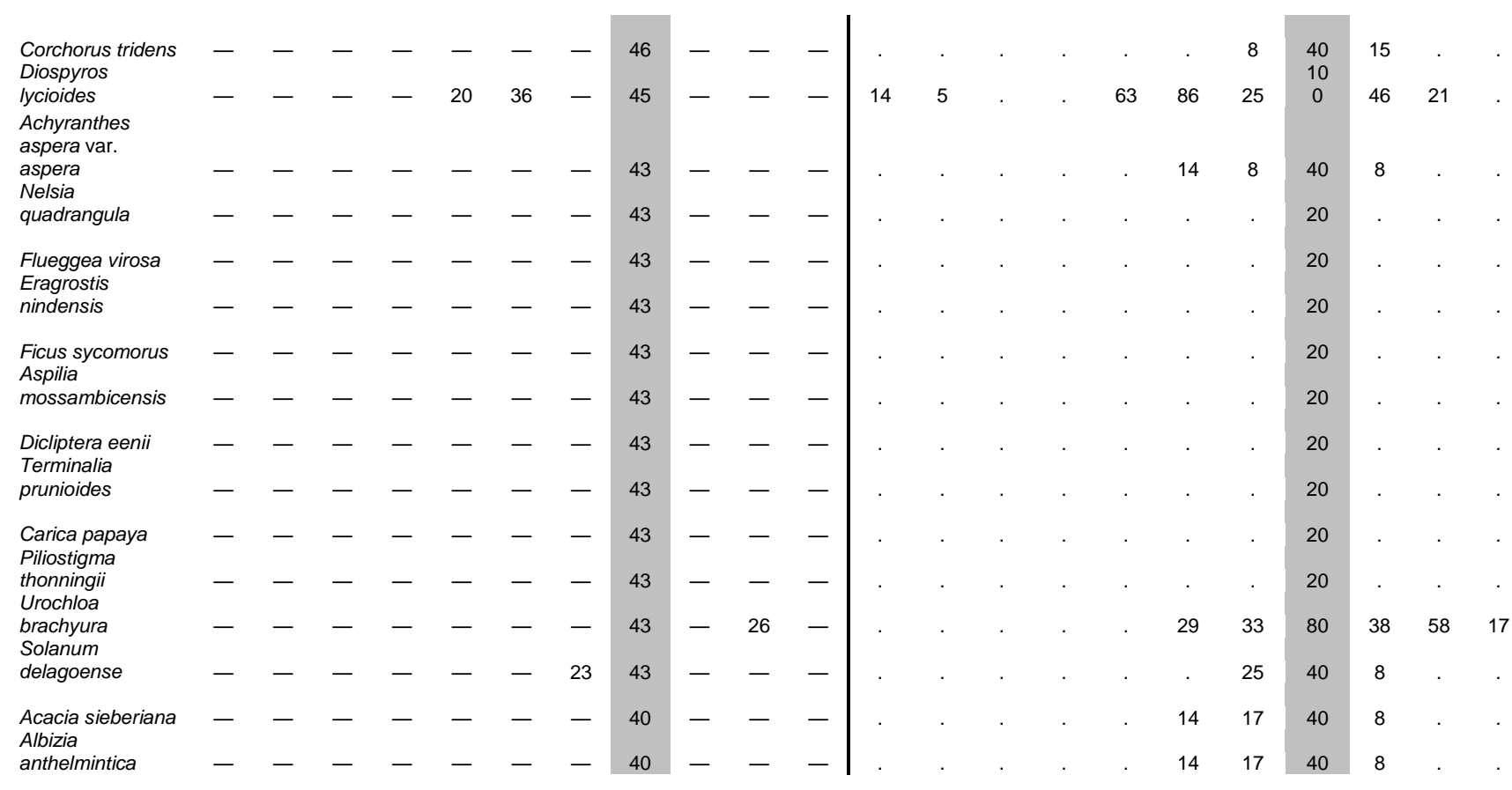

Association 7: Acacia erioloba-Schinziophyton rautanenii association

\begin{tabular}{llllllllllllll|}
$\begin{array}{l}\text { Vangueria } \\
\text { cyanescens }\end{array}$ & - & - & - & - & - & - & - & - & 52 & - & - \\
$\begin{array}{l}\text { Indigofera } \\
\text { flavicans }\end{array}$ & - & - & - & - & - & - & - & - & 44 & 25 & - \\
Acacia erioloba & - & - & - & - & - & 27 & - & - & 41 & 25 & -
\end{tabular}

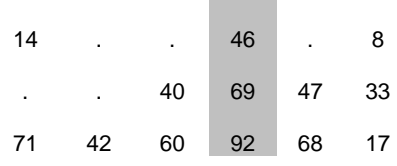

Association 8: Acacia erioloba-Combretum collinum association

\begin{tabular}{|c|c|c|c|c|c|c|c|c|c|c|}
\hline $\begin{array}{l}\text { Eragrostis } \\
\text { lehmanniana }\end{array}$ & - & - & - & - & - & - & - & - & - & 49 \\
\hline Acanthosicyos & & & & & & & & & & \\
\hline $\begin{array}{l}\text { naudinianus } \\
\text { Croton }\end{array}$ & - & - & - & - & - & - & - & - & - & 46 \\
\hline $\begin{array}{l}\text { gratissimus } \\
\text { Commiphora }\end{array}$ & - & - & - & - & - & - & - & - & - & 44 \\
\hline $\begin{array}{l}\text { africana } \\
\text { Oxygonum }\end{array}$ & - & - & - & - & - & - & - & - & - & 42 \\
\hline alatum & - & - & - & - & - & - & - & - & 24 & 42 \\
\hline
\end{tabular}

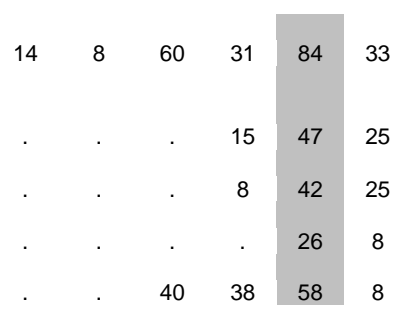

Association 9: Pterocarpus angolensis-Guibourtia coleosperma association

\begin{tabular}{|c|c|c|c|c|c|c|c|c|c|c|}
\hline $\begin{array}{l}\text { angolensis } \\
\text { Cyperus }\end{array}$ & - & - & - & - & - & - & - & - & - & - \\
\hline margaritaceus & - & - & - & - & - & - & - & - & - & - \\
\hline Eragrostis pallens & - & 一 & 一 & - & - & - & 一 & 一 & - & - \\
\hline $\begin{array}{l}\text { Burkea africana } \\
\text { Evolvulus }\end{array}$ & - & - & - & - & - & - & - & - & - & 一 \\
\hline alsinoides & - & - & 一 & - & - & - & - & - & - & - \\
\hline $\begin{array}{l}\text { Ochna pulchra } \\
\text { Tephrosia }\end{array}$ & - & - & - & - & - & - & - & - & - & 19 \\
\hline $\begin{array}{l}\text { lupinifolia } \\
\text { Combretum }\end{array}$ & 一 & 一 & - & - & - & 一 & 一 & 一 & - & 一 \\
\hline $\begin{array}{l}\text { psidioides } \\
\text { Stylosanthes }\end{array}$ & - & - & - & - & - & - & - & - & - & - \\
\hline fruticosa & - & - & - & - & - & - & - & - & - & - \\
\hline $\begin{array}{l}\text { Xenostegia } \\
\text { tridentata subsp. } \\
\text { angustifolia }\end{array}$ & - & - & - & - & - & 一 & - & - & - & 24 \\
\hline $\begin{array}{l}\text { Indigofera filipes } \\
\text { Polygala }\end{array}$ & - & - & - & - & - & - & - & - & - & - \\
\hline schinziana & - & - & - & - & - & - & - & - & - & - \\
\hline $\begin{array}{l}\text { Baphia } \\
\text { massaiensis } \\
\text { subsp. obovata }\end{array}$ & & & & & & & & & & \\
\hline var. obovata & - & - & - & - & - & - & - & - & - & 20 \\
\hline Aristida stipitata & - & - & - & - & - & - & - & - & - & - \\
\hline
\end{tabular}




\begin{tabular}{|c|c|c|c|c|c|c|c|c|c|c|c|c|c|c|c|c|c|c|c|c|c|c|}
\hline $\begin{array}{l}\text { Tephrosia } \\
\text { purpurea subsp. } \\
\text { leptostachya var. } \\
\text { pubescens } \\
\text { Rhynchosia }\end{array}$ & - & - & - & - & - & - & - & - & - & - & 65 & . & . & . & . & . & . & . & . & . & 16 & 58 \\
\hline venulosa & - & - & - & - & - & - & - & - & - & - & 65 & . & . & . & . & . & . & 8 & . & . & 21 & 67 \\
\hline $\begin{array}{l}\text { Baikiaea plurijuga } \\
\text { Ozoroa }\end{array}$ & - & - & - & - & - & - & - & - & - & 25 & 64 & . & . & . & . & . & . & . & . & . & 32 & 67 \\
\hline okavangensis & - & - & - & - & - & - & - & - & - & - & 63 & . & . & . & . & . & . & . & . & . & . & 42 \\
\hline $\begin{array}{l}\text { Securidaca } \\
\text { longepedunculata }\end{array}$ & - & - & - & - & - & - & - & - & - & - & 63 & . & . & . & . & . & . & . & . & . & . & 42 \\
\hline $\begin{array}{l}\text { Melinis repens } \\
\text { subsp. grandiflora } \\
\text { Strychnos }\end{array}$ & - & - & - & - & - & - & - & - & - & - & 62 & . & . & . & . & . & . & . & . & . & 11 & 50 \\
\hline $\begin{array}{l}\text { cocculoides } \\
\text { Megaloprotachne }\end{array}$ & - & - & - & - & - & - & - & - & - & - & 59 & . & . & . & . & . & . & . & . & . & 16 & 50 \\
\hline $\begin{array}{l}\text { albescens } \\
\text { Combretum }\end{array}$ & - & - & - & - & - & - & - & - & - & - & 59 & . & . & . & . & . & . & . & . & . & 5 & 42 \\
\hline zeyheri & - & - & - & - & - & - & - & - & - & - & 58 & . & . & . & . & . & . & . & . & 15 & 16 & 58 \\
\hline $\begin{array}{l}\text { Stipagrostis } \\
\text { uniplumis var. } \\
\text { uniplumis }\end{array}$ & - & - & - & - & - & - & - & - & - & - & 56 & . & . & . & . & . & 29 & . & . & . & 26 & 67 \\
\hline $\begin{array}{l}\text { Gardenia } \\
\text { brachythamnus }\end{array}$ & - & - & - & - & - & - & - & - & - & - & 56 & . & . & . & . & . & . & . & . & . & . & 33 \\
\hline $\begin{array}{l}\text { Fadogia thamnus } \\
\text { Lannea }\end{array}$ & - & - & - & - & - & - & - & - & - & - & 56 & . & . & . & . & . & . & . & . & . & . & 33 \\
\hline gossweileri & - & - & - & - & - & - & - & - & - & - & 56 & . & . & . & . & . & . & . & . & . & . & 33 \\
\hline $\begin{array}{l}\text { Heteropogon } \\
\text { melanocarpus } \\
\text { Raphionacme }\end{array}$ & - & - & - & - & - & - & - & - & - & - & 56 & . & . & . & . & . & . & . & . & . & . & 33 \\
\hline $\begin{array}{l}\text { lanceolata } \\
\text { Vernonia }\end{array}$ & - & - & - & - & - & - & - & - & - & - & 55 & . & . & . & . & . & . & . & . & . & 11 & 42 \\
\hline $\begin{array}{l}\text { poskeana } \\
\text { Guibourtia }\end{array}$ & - & - & - & - & - & - & - & - & - & - & 55 & . & . & . & . & . & . & . & . & 8 & 16 & 50 \\
\hline $\begin{array}{l}\text { coleosperma } \\
\text { Chamaecrista }\end{array}$ & - & - & - & - & - & - & - & - & - & 24 & 54 & . & . & . & . & . & . & . & . & . & 26 & 50 \\
\hline biensis & - & - & - & - & - & - & - & - & - & 24 & 54 & . & . & . & . & . & . & . & $\cdot$ & . & 26 & 50 \\
\hline $\begin{array}{l}\text { Commiphora } \\
\text { tenuipetiolata } \\
\text { Aristida }\end{array}$ & - & - & - & - & - & - & - & - & - & - & 52 & . & . & . & . & . & . & . & . & 15 & . & 42 \\
\hline meridionalis & - & - & - & - & - & - & - & - & - & - & 52 & . & . & . & . & . & . & . & . & . & 16 & 42 \\
\hline $\begin{array}{l}\text { Cyphostemma } \\
\text { sandersonii }\end{array}$ & - & - & - & - & - & - & - & - & - & - & 51 & . & . & . & . & . & . & . & . & . & 5 & 33 \\
\hline $\begin{array}{l}\text { Diospyros } \\
\text { chamaethamnus } \\
\text { Strychnos }\end{array}$ & - & - & - & - & - & - & - & - & - & - & 51 & . & . & . & . & . & . & . & . & . & 5 & 33 \\
\hline pungens & - & - & - & - & - & - & - & - & - & - & 48 & . & . & . & . & . & . & . & . & . & . & 25 \\
\hline $\begin{array}{l}\text { Entada arenaria } \\
\text { Justicia protracta } \\
\text { subsp. rhode- }\end{array}$ & - & - & - & - & - & - & - & - & - & - & 48 & . & . & . & . & . & . & . & . & . & . & 25 \\
\hline $\begin{array}{l}\text { siana } \\
\text { Tephrosia } \\
\text { cephalantha var. }\end{array}$ & - & - & - & - & - & - & - & - & - & - & 48 & . & . & . & . & . & . & . & . & . & . & 25 \\
\hline $\begin{array}{l}\text { decumbens } \\
\text { Chamaesyce } \\
\text { neo- }\end{array}$ & - & - & - & - & - & - & - & - & - & - & 48 & . & . & . & . & . & . & . & . & . & . & 25 \\
\hline $\begin{array}{l}\text { polycnemoides } \\
\text { Tristachya }\end{array}$ & - & - & - & - & - & - & - & - & - & - & 48 & . & . & . & . & . & . & . & . & . & . & 25 \\
\hline superba & - & - & - & - & - & - & - & - & - & - & 47 & . & . & . & . & . & . & . & . & . & 11 & 33 \\
\hline $\begin{array}{l}\text { Hermannia eenii } \\
\text { Limeum }\end{array}$ & - & - & - & - & - & - & - & - & - & - & 47 & . & . & . & . & . & . & . & . & . & 11 & 33 \\
\hline fenestratum & - & - & - & - & - & - & - & - & - & 31 & 47 & . & . & . & . & . & . & . & . & 23 & 42 & 58 \\
\hline $\begin{array}{l}\text { Melinis repens } \\
\text { subsp. repens }\end{array}$ & - & - & - & - & - & - & - & - & - & 26 & 47 & . & . & . & . & . & . & . & . & . & 26 & 42 \\
\hline $\begin{array}{l}\text { Schinziophyton } \\
\text { rautanenii }\end{array}$ & - & - & - & - & - & - & - & - & 26 & 20 & 46 & . & . & . & . & . & . & . & . & 38 & 32 & 58 \\
\hline Perotis patens & - & - & - & - & - & - & - & - & - & - & 45 & . & . & . & . & . & 43 & . & 20 & 23 & 16 & 67 \\
\hline $\begin{array}{l}\text { Ozoroa schinzii } \\
\text { Ceratotheca }\end{array}$ & - & - & - & - & - & - & - & - & - & 32 & 44 & . & . & . & . & . & . & . & . & . & 32 & 42 \\
\hline sesamoides & - & - & - & - & - & - & - & - & - & - & 43 & . & . & . & . & . & . & . & . & . & 5 & 25 \\
\hline $\begin{array}{l}\text { Indigofera trita } \\
\text { subsp. subulata }\end{array}$ & - & - & - & - & - & - & - & - & - & - & 43 & . & . & . & $\cdot$ & . & . & . & . & . & 5 & 25 \\
\hline Tricholaena & & & & & & & & & & & 43 & . & . & . & . & . & . & . & . & 8 & 11 & 33 \\
\hline
\end{tabular}




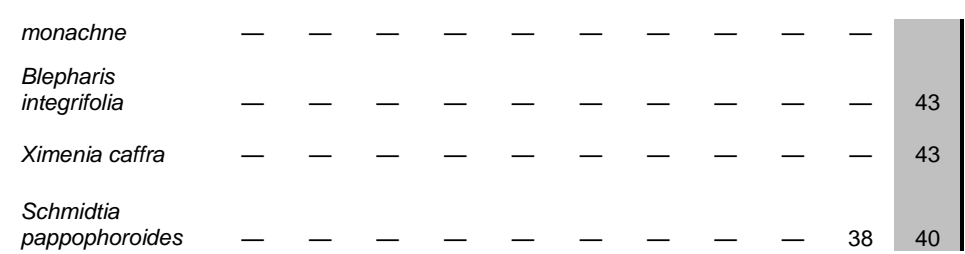

Common diagnostic species of two or more associations:

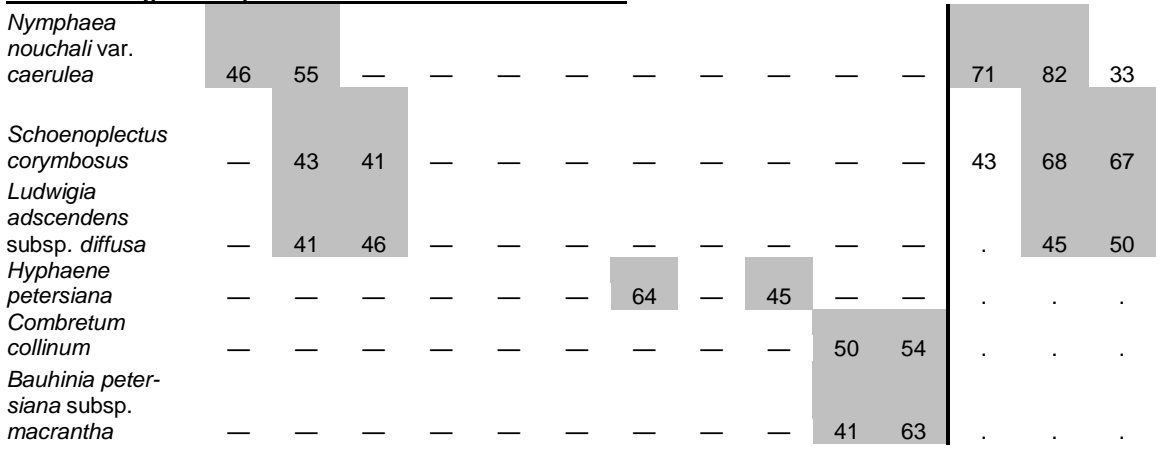

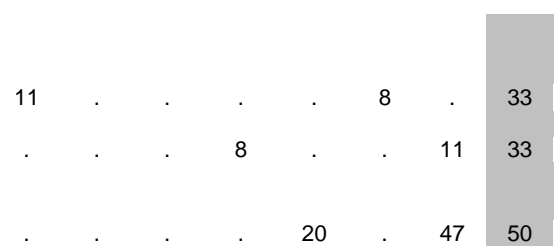

13

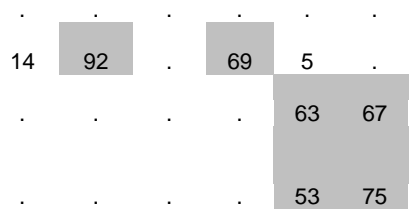

association. A total of 33 species have been observed in this association, with on average 11.4 species per $1000 \mathrm{~m}^{2}$.

This association is formed along the river banks of the Okavango River (Fig. 6a). Depending on the steepness of the bank, the abundance of the reed Phragmites australis varies. On shallow banks, which are largely inundated, extensive reed banks can be formed, which form important habitats for fish spawning and nesting sites for birds alike (Hines 1985, Høberg et al. 2002, Junk et al. 1989, King et al. 2003, Merron \& Bruton 1995). Generally, the base of this association is always inundated during the high-flow season (January to June), whilst in extreme high flow years this association could be well over two meters deep under water (Fig. 7a).

\subsection{Paratheria prostrata- Nymphaea nouchali association of the lakes and seasonally flooded floodplains}

This association is characterized by the diagnostic species Nymphaea nouchali var. caerulea, Eleocharis acutangula, Oryza longistaminata, Utricularia stellaris, Nymphaea lotus, Paratheria prostrata, Ipomoea aquatica, Schoenoplectus corymbosus, Panicum coloratum and Ludwigia adscendens subsp. diffusa. These are constantly associated by Persicaria senegalensis, Aeschynomene fluitans and Oryzidium barnardii. Twentytwo relevés have been classified into this association. A total of 50 species have been observed in this association, with on average 9.9 species per $1000 \mathrm{~m}^{2}$.
These floodplains occur adjacent to the river banks, but lower than the banks at a range of 0 to $2 \mathrm{~m}$ above river level (average $1.1 \mathrm{~m}$ above river level). They are generally shielded from the river by the somewhat higher river banks. This results in the floodplains drying up completely during the low-flow season (June to December) (Fig. 6b and c, Fig. 7b), and are only flooded once the river level rises above certain low points in the sand banks. Further inland, some old oxbow lakes in the floodplains also form part of this system. Depending on the depth, a variety of aquatic plants occur here. The shallow fringes are dominated by Paratheria prostrata, a fine, emergent grass species easily confused with Cynodon dactylon. It is likely that Hines mistook this species for Cynodon dactylon in his description of the "perched river terrace wetlands" in the Kavango region near Ekongoro (Hines 1993). In ca 50 to $80 \mathrm{~cm}$ deep water, Nymphaea nouchali and Schoenoplectus corymbosus dominate, with $N$. lotus occurring only in 80 to 120 $\mathrm{cm}$ deep water. Dense stands of Oryza longistaminata and/or Oryzidium barnardii occasionally occur in these water depth ranges (Fig. 6d). Deeper water is generally clear of emerging aquatic plants, but a variety of submerged plants (e.g. Utricularia stellaris, Lagarosiphon spp. and Rotala spp.) can be observed here.

\subsection{Tacazzea apiculata-Chrys- opogon nigritanus fringe grassland association}

This association is characterized by the diagnostic species Tacazzea apiculata,
Heliotropium baclei, Ludwigia adscendens subsp. diffusa, Crinum caroloschmidtii and Schoenoplectus corymbosus. These are constantly associated by Chrysopogon nigritanus, Paratheria prostrate and Oryzidium barnardii. Six relevés have been classified into this association. A total of 41 species have been observed in this association, with on average 11.0 species per $1000 \mathrm{~m}^{2}$.

These grasslands occur on the fringes of the Paratheria prostrata-Nymphaea nouchali floodplains, at on average $1.8 \mathrm{~m}$ above the level of the river. Depending on the height of the flood, the Tacazzea apiculata-Chrysopogon nigritanus fringe grasslands can easily be flooded (Fig. 7c), and thus share a large number of species (either as remnants from recent high floods, or as potential vegetation in seed banks and/or as aquatic geophytes) with the previous association. Conspicuous, however, is the high abundance of Chrysopogon nigritanus in this association, as well as several hydrophytic, but nonaquatic species like Tacazzea apiculata and Heliotropium baclei (Fig. 6e).

\section{Sporobolus ioclados- Willkom- mia sarmentosa saline grasslands of the Hakusembe floodplains}

This association is characterized by the diagnostic species Willkommia sarmentosa, Sporobolus ioclados, Sesuvium sesuvioides, Sporobolus albicans, Monandrus squarrosus, Cyperus schinzii, Cyperus longus and Brachiaria xantholeuca. Nine relevés have been classified into this association. A total of 33 species have 
Table 3: Average soil and habitat characteristics for the various associations.

\begin{tabular}{|c|c|c|c|c|c|c|c|c|c|c|c|c|c|c|}
\hline Association & $\begin{array}{l}\text { Approx. height } \\
\text { above river [m] }\end{array}$ & $\mathrm{pHw}$ & $\begin{array}{c}E C w \\
\mu S / c m\end{array}$ & $\begin{array}{c}\text { OC } \\
\% \\
\end{array}$ & $\begin{array}{c}\mathrm{P} \\
\mathrm{ppm}\end{array}$ & $\begin{array}{l}\text { K-Exch } \\
\text { me/ 100g }\end{array}$ & $\begin{array}{l}\text { Ca-Exch } \\
\text { me/ 100g }\end{array}$ & $\begin{array}{l}\text { Mg-Exch } \\
\text { me/ 100g }\end{array}$ & $\begin{array}{l}\text { Na-Exch } \\
\text { me/ 100g }\end{array}$ & $\begin{array}{c}\text { CEC } \\
\mathrm{me} / 100 \mathrm{~g}\end{array}$ & Texture & $\begin{array}{c}\text { Sand } \\
\% \\
\end{array}$ & $\begin{array}{c}\text { Silt } \\
\% \\
\end{array}$ & $\begin{array}{c}\text { Clay } \\
\% \\
\end{array}$ \\
\hline 1.1 & 1.00 & 4.93 & 30 & 0.32 & 0.0 & 0.03 & 0.66 & 0.13 & 0.01 & 0.79 & Sand & 93.8 & 3.2 & 3 \\
\hline 1.2 & 0.91 & 5.61 & 110 & 1 & 4.0 & 1.08 & 12.84 & 4.88 & 0.24 & 18.79 & Silty clay & 19.85 & 31.05 & 49.1 \\
\hline 1.3 & 1.83 & 5.26 & 79 & 0.35 & 7.9 & 1.12 & 2.1 & 0.6 & 0.11 & 3.82 & Loamy sand & 79.1 & 12.2 & 8.6 \\
\hline 2 & 4.22 & 9.77 & 457 & 0.09 & 8.1 & 0.15 & 3.25 & 0.34 & 5.31 & 9.05 & Loamy sand & 81.4 & 11.5 & 7.1 \\
\hline 3 & 2.67 & 5.36 & 32 & 0.67 & 0.0 & 0.11 & 4.8 & 0.76 & 0.04 & 5.67 & Clay loam & 38.4 & 22.9 & 38.7 \\
\hline 4 & 2.71 & 5.65 & 16 & 0.20 & 3.0 & 0.08 & 0.84 & 0.24 & 0.10 & 1.10 & Sand & 93.2 & 4.05 & 2.75 \\
\hline 5 & 6.25 & 6.54 & 63 & 0.26 & 6.2 & 0.24 & 2.33 & 0.49 & 0.22 & 3.27 & Loamy sand & 84.9 & 4.1 & 10.9 \\
\hline 6 & 9.00 & 5.65 & 16 & 0.19 & 3.0 & 0.08 & 0.83 & 0.24 & 0.09 & 1.10 & Sand & 93.2 & 4.1 & 2.8 \\
\hline 7 & 6.38 & 6.69 & 174 & 0.15 & 4.6 & 0.11 & 0.62 & 0.36 & 0.03 & 0.69 & Sand & 94.9 & 2.7 & 2.5 \\
\hline 8 & 33.84 & 6.37 & 41 & 0.35 & 5.4 & 0.14 & 1.44 & 0.38 & 0.05 & 1.9 & Sand & 95.5 & 2.5 & 2.1 \\
\hline 9 & 49.83 & 6.42 & 45 & 0.24 & 5.0 & 0.11 & 1.03 & 0.31 & 0.06 & 1.47 & Sand & 95.6 & 3.0 & 1.5 \\
\hline
\end{tabular}




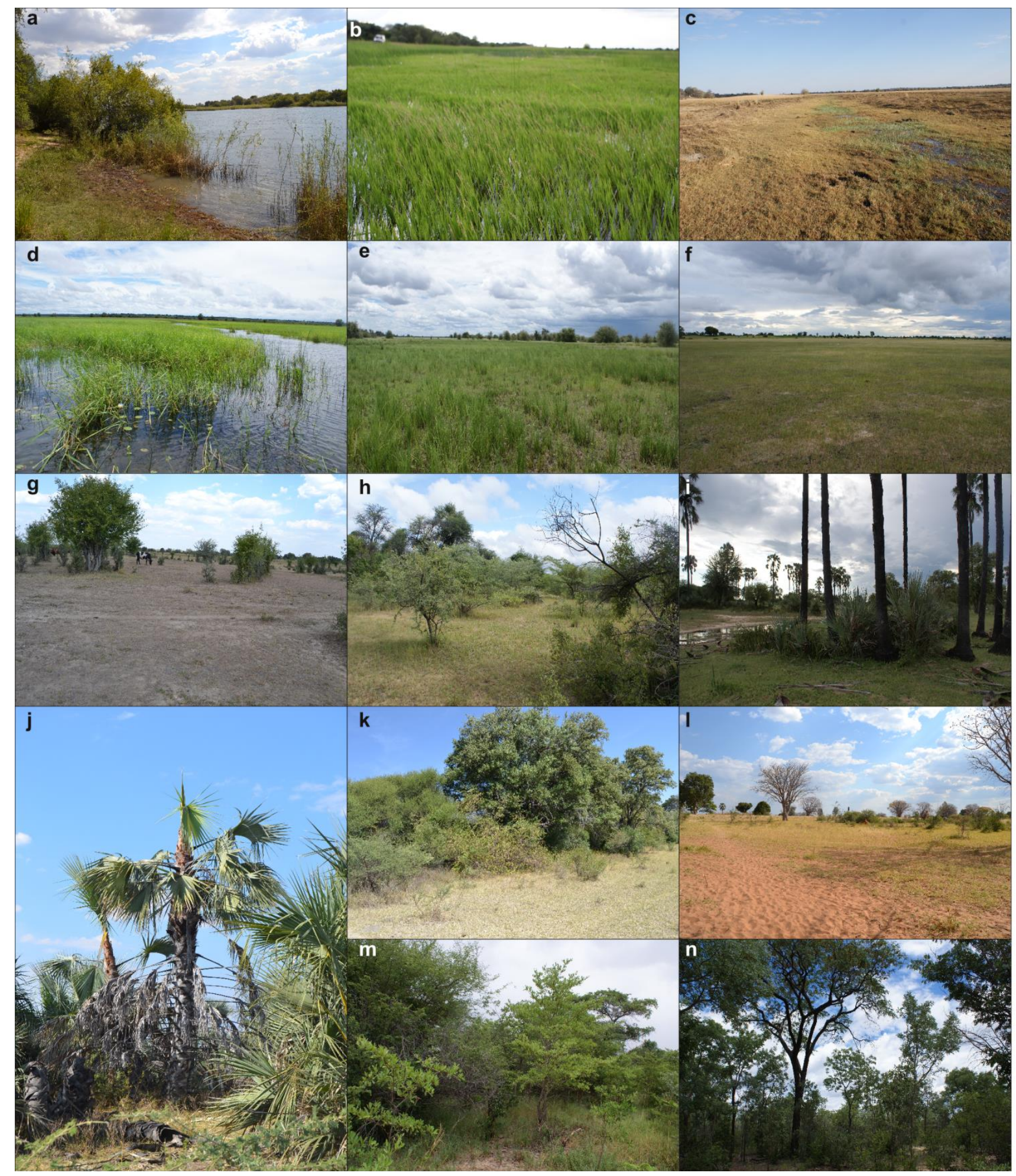

Fig. 6: Photos of typical relevés of the various associations identified. a: Mimosa pigra-Phragmites australis association; b, c \& d: Paratheria prostrata-Nymphaea nouchali association: b and c of a Paratheria prostrata-dominated floodplain in February and July 2013 respectively; d of a floodplain dominated by Oryza longistaminata with occasional Nymphaea nouchaIi; e: Tacazzea apiculata- Chrysopogon nigritanus association; f: Sporobolus ioclados- Willkommia sarmentosa association; g: Chrysopogon nigritanus- Gardenia volkensii association; h: Terminalia sericea- Combretum imberbe association; i: Acacia tortilis-Hyphaene petersiana association; j: Hyphaene petersiana palms which have been tapped for palm wine; k: Acacia sieberiana-Ficus association; I: Acacia erioloba-Schinziophyton rautanenii association; m: Acacia erioloba-Combretum collinum association; n: Pterocarpus angolensis-Guibourtia coleosperma association. 

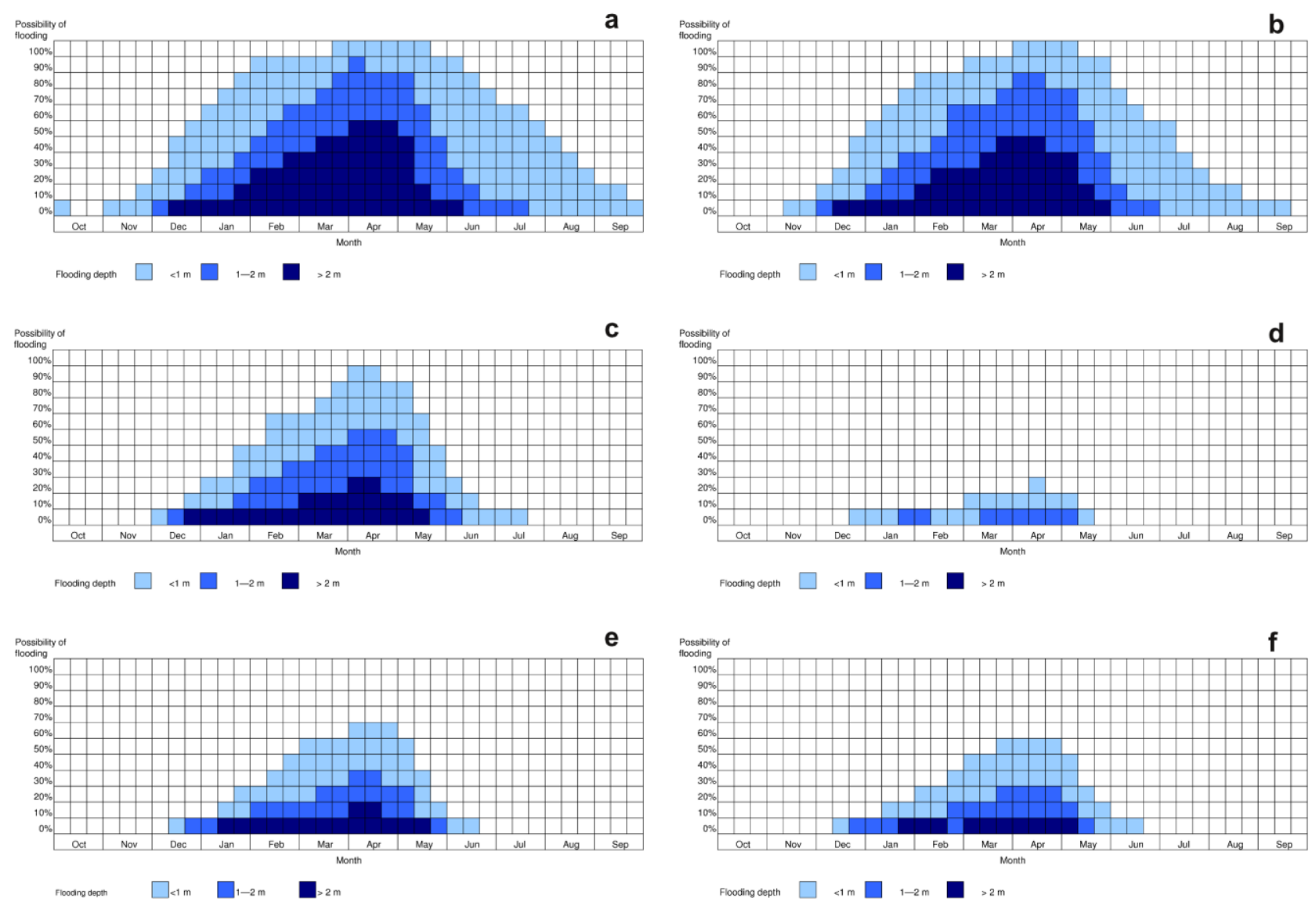

Fig. 7: Flood regimes of the various wetland and floodplain associations. a: Mimosa pigra-Phragmites australis association; b: Paratheria prostrata-Nymphaea nouchali association; c: Tacazzea apiculata- Chrysopogon nigritanus association; d: SporoboIus ioclados- Willkommia sarmentosa association; e: Chrysopogon nigritanus- Gardenia volkensii association; f: Terminalia sericea- Combretum imberbe association.

been observed in this association, with on average 7.0 species per $1000 \mathrm{~m}^{2}$.

The extensive Hakusembe grasslands are an anomaly in the otherwise mineralpoor, sand dominated landscapes of the Okavango River, as the soils here have a very high $\mathrm{pH}$ (9.8 in the topsoil, 10.8 in the B-horizon, as measured in water) as well as a high sodium content (5.3 $\mathrm{me} / 100 \mathrm{~g}$ in the topsoil, $19.0 \mathrm{me} / 100 \mathrm{~g}$ in the B-horizon) (Table 3). These saline soils thus support only a short, open grassland, very similar in composition and structure to the oshonas of the Cuvelai delta in Central-Northern Namibia (Kangombe 2010) (Fig. 6f). These grassy plains are on average $4.0 \mathrm{~m}$ above the river level (range 1 to $5 \mathrm{~m}$ ), and are flooded only in two out of ten years, to a very shallow water depth of just under a meter. Only in extreme floods (one out of ten years), the water level could rise to over 1 m (Fig. 7d). Similar vegetation has been observed east of Rundu at Kayengona.

\section{Chrysopogon nigritanus- Gar- denia volkensii floodplain wood- lands}

This association is characterized by the diagnostic species Geigeria nianganensis, Aristida junciformis subsp. junciformis, Nesaea rigidula, Eragrostis annulata and Brachiaria humidicola, which are constantly accompanied by Gardenia volkensii subsp. spatulifolia, Eragrostis trichophora, Cynodon dactylon, Chrysopogon nigritanus, Diospyros lycioides and Combretum imberbe. Seven relevés have been classified into this association. A total of 52 species have been observed in this association, with on average 13.5 species per $1000 \mathrm{~m}^{2}$.

Directly following the Tacazzea apiculata-Chrysopogon nigritanus association in the catena, this association is on average $2.5 \mathrm{~m}$ above the river level (range 1 $5 \mathrm{~m}$ ). Flooding occurs regularly in six out of 10 years, but only in two out of 10 years the flooding level is deeper than $2 \mathrm{~m}$ (Fig. 7e). This results in a limited devel- opment of the phanerophytic layer, with Gardenia volkensii, as a known wetland species, dominating (Fig. 6g). Most other tree species (with the exception of Combretum imberbe) do not seem to be able to tolerate prolonged waterlogged soil conditions (anaerobic soil conditions).

\section{Terminalia sericea-Combretum imberbe wooded floodplains}

This association is characterized by the diagnostic species Euclea undulata, Andropogon schirensis, Asparagus racemosus, Acacia hebeclada, Kyllinga alba, Pogonarthria squarrosa, Ehretia rigida, Combretum imberbe and Acacia luederitzii. These are constantly associated by Diospyros lycioides, Terminalia sericea, Peltophorum africanum, Gardenia volkensii subsp. spatulifolia, Eragrostis trichophora, Cynodon dactylon, Acacia erioloba, Jasminum fluminense, Grewia flavescens, Acacia fleckii, Ziziphus mucronata, Perotis patens, Ocimum americanum var. americanum 
and Digitaria seriata. Seven relevés have been classified into this association. A total of 91 species have been observed in this association, with on average 25.6 species per $1000 \mathrm{~m}^{2}$.

Again following the Chrysopogon nigitianus-Gardenia volkensii association in the catena, the Terminalia sericeaCombretum imberbe association forms a dense bushland, even thicket (Fig. 6h), on extensive sandy floodplains. These are on average $2.7 \mathrm{~m}$ above the river level, with a range of 1-4 m. Flooding happens only every second year, at a relatively low flooding level and short duration (Fig. 7f). This, coupled to the sandy soils with better drainage, allows other phanerophytic species to establish. Due to the varying height above the river level, and thus varying flooding regime, this association displays also a rather large internal variation. The denser stands of Gardenia volkensii seem to be correlated with the lower, more flood-prone areas of these floodplains, whilst in the higher parts, this species is gradually replaced by Terminalia sericea and Acacia fleckii. Of note are that both A. fleckii and A. erubescens have been found on these floodplains and might have been confused during surveys, due to their close resemblance in growth form and bark colour. Likewise, all three subspecies of Acacia hebeclada (A. hebeclada subsp. hebeclada, A. hebeclada subsp. chobiensis and A. hebeclada subsp. tristris) have been identified here. At the same time, many plants of this species were found without pods, thus making identification to subspecies level impossible.

\section{Acacia tortilis-Hyphaene peter- siana palm plains}

This association is characterized by the diagnostic species Hyphaene petersiana, Acacia tortilis, Ipomoea adenioides, Chloris virgata, Eragrostis trichophora, Sansevieria aethiopica, Pergularia daemia, Indigofera rautanenii and Urochloa oligotricha. These are constantly associated by Cynodon dactylon, Combretum imberbe, Ocimum americanum var. americanum, Dichrostachys cinerea, Gymnosporia senegalensis, Grewia flavescens and Acacia erioloba. Twelve relevés have been classified into this association. A total of 125 species have been observed in this association, with on average 24.2 species per $1000 \mathrm{~m}^{2}$.

These characteristic palm plains (Fig. 6i) occasionally occur as prominent fringes along floodplains with somewhat higher salinity, or as transition zone between the Terminalia sericea- Combretum imberbe and the Acacia eriolobaSchinziophyton rautanenii associations. They are on average $6.25 \mathrm{~m}$ above the river level (with a range of 3-9 m), and are generally beyond the flooding level. Anecdotal evidence suggests that palm trees (e.g. Phoenix dactylifera, but also Hyphaene petersiana) like "wet feet and a hot head", i.e. standing on the fringes of floodplains, beyond the reach of flooding, but within easy reach of ground water.

Hyphaene petersiana is establishing very well in adjacent vegetation associations, in particular the Terminalia sericea-Combretum imberbe and the Acacia erioloba-Schinziophyton rautanenii associations. This makes the edges of the Acacia tortilis-Hyphaene petersiana association rather fuzzy - both in the landscape as well as in the classification. The palms are not as extensively utilized as in the Central North (in particular the Oshana Region) (Cunningham et al. 1992, Strohbach et al. 2002, Sullivan et al. 1995), but some basketry is done in Kavango (Rössing Foundation 2002). Some palm wine tapping has also been observed here (Fig. 6j).

\section{Acacia sieberiana-Ficus thickets on steep banks}

This association is characterized by the diagnostic species Ocimum gratissimum, Acrotome inflata, Senna occidentalis, Jasminum fluminense, Hibiscus caesius, Abutilon angulatum, Senna obtusifolia, Setaria verticillata, Tragia okanyua, Panicum maximum, Zehneria marlothii, Monechma divaricatum, Hermannia guerkeana, Helinus integrifolius, Asparagus nelsii, Gymnosporia senegalensis, Aerva leucura, Sida ovata, Ficus thonningii, Corchorus tridens, Diospyros lycioides, Achyranthes aspera var. aspera, Terminalia prunioides, Piliostigma thonningii, Nelsia quadrangula, Flueggea virosa, Ficus sycomorus, Eragrostis nindensis, Dicliptera eenii, Carica papaya, Aspilia mossambicensis, Urochloa brachyura, Solanum delagoense, Albizia anthelmintica and Acacia sieberiana. These are constantly associated by Ziziphus mucronata, Grewia flavescens, Dichrostachys cinerea, Combretum imberbe, Spermacoce senensis, Peltophorum africanum, Eragrostis lehmanniana, Cynodon dactylon and Acacia eriol$o b a$. Five relevés have been classified into this association. A total of 94 species have been observed in this association, with on average 35.2 species per $1000 \mathrm{~m}^{2}$.

This thicket association occurs on moderately steep embankments between the floodplains and the valley bottom. These embankments are however generally limited in occurrence and extent, thus this association is also rather limited in extent. The soils, albeit sandy, are shallow on a calcrete (or calcareous sandstone) sublayer. These banks are out of flooding reach, on average $9 \mathrm{~m}$ above the river level. The floristic composition of this association has strong resemblances with Karstveldtype thickets, with Terminalia prunioides, Flueggea virosa, Panicum maximum, Euclea undulata, Aerva leucura and other species from that biome (Fig. 6k). Very conspicuous are the yellow-barked Acacia sieberiana trees (although not limited to this association), as well as big trees of either Ficus sycomorus or F. thoningii.

\section{Acacia erioloba-Schinziophyton rautanenii disturbed woodlands of the valley bottom}

This association is characterized by the diagnostic species Vangueria cyanescens, Hyphaene petersiana, Indigofera flavicans and Acacia erioloba, constantly associated by Cynodon dactylon, Terminalia sericea, Grewia flavescens, Dichrostachys cinerea, Ziziphus mucronata, Eragrostis trichophora and Acrotome spp. Thirteen relevés have been classified into this association. A total of 143 species have been observed in this association, with on average 28.3 species per $1000 \mathrm{~m}^{2}$. 
This association represents the valley bottom - basically that ecosystem in which most settlements are located, and most farming activities take place. With the ever increasing population (Mendelsohn \& el Obeid 2003), well over $90 \%$ of this association has been ploughed, with the remainder severely deforested and degraded. Only remnants of the original vegetation are remaining between homesteads and fields (Fig. 61). This results in an incomplete picture of the potential species composition, as is evident in the relatively low species richness of the association, and also low internal variability (Fig. 8).

\section{Acacia erioloba-Combretum collinum bushlands of the terrace slope}

This association is characterized by the diagnostic species Combretum collinum, Eragrostis lehmanniana, Acanthosicyos naudinianus, Croton gratissimus, Oxygonum alatum, Commiphora africana and Bauhinia petersiana subsp. macrantha. These are constantly associated by Terminalia sericea, Grewia flavescens, Acacia erioloba, Digitaria seriata, Dichrostachys cinerea, Urochloa brachyura, Spermacoce senensis, Peltophorum africanum, Acacia fleckii and Searsia tenuinervis. Nineteen relevés have been classified into this association. A total of 220 species have been observed in this association, with on average 38.3 species per $1000 \mathrm{~m}^{2}$.

As the richer alluvial soils of the valley bottom are covered by the nutrient-poor Kalahari sand deposits, the vegetation changes from the Acacia eriolobaSchinziophyton rautanenii association to the Acacia erioloba-Combretum collinum association of the terrace slopes. As the sand gets deeper, more and more sand vegetation takes over, with a dense understory of Terminalia sericea, various Combretum species, Bauhinia petersiana and Acacia fleckii (Fig. 6m). This association is common along the slopes of the Okavango River terrace, but is also found along the slopes to the various omirimbi contributing to the Okavango (e.g. Mpungu and Mpuku).

The border between this association and the valley bottom is quite distinctively visible on aerial photographs through a change in soil colour, and generally an end to especially the older fields. This association is often cleared for new fields, but at the same time, numerous of these

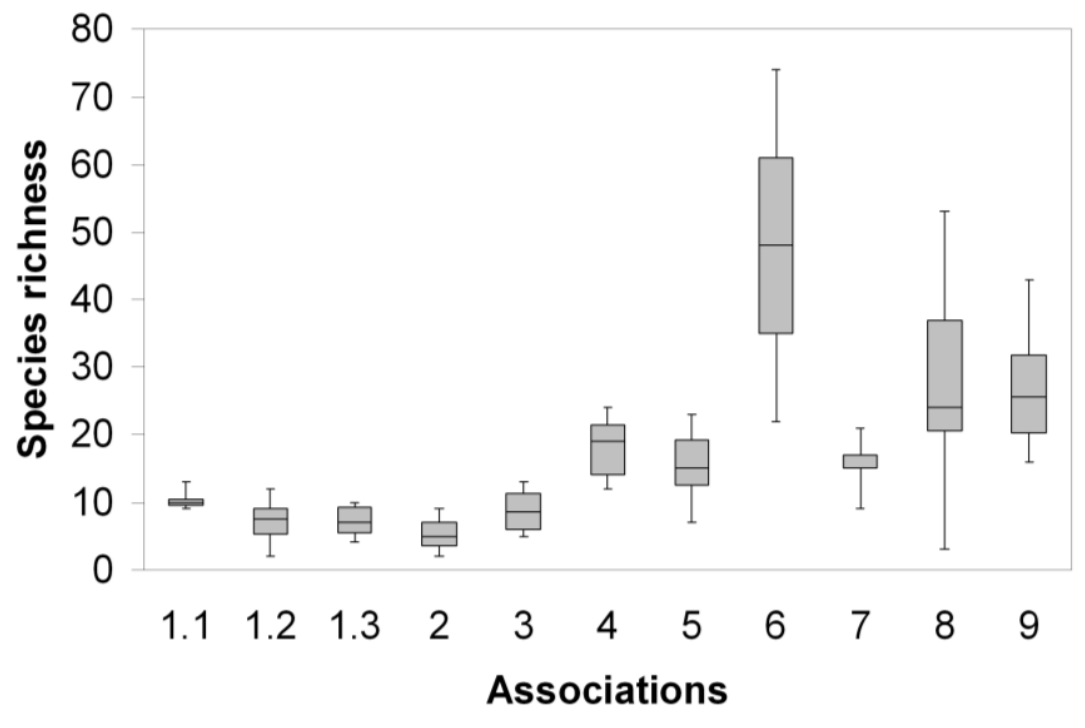

Fig.8: Graphic comparison of the species richness of the different associations.

newly cleared fields are not cropped due to their low productivity.

A similar vegetation association has been described for the Alex Muranda LDC (former Mile 46 LDC) in centralsouthern Kavango, the Acacia fleckiiTerminalia sericea association (Strohbach \& Petersen 2007). This association is thought to be a secondary climax after extensive fire damage to the Kavango woodlands. Exactly how the Acacia fleckii-Terminalia sericea association relates to the Acacia erioloba-Combretum collinum association has not been established. The Acacia erioloba-Combretum collinum association, though, is seen as an ecotonal association between the Pterocarpus angolensis-Guibourtia coleosperma association of the Kavango woodlands and the Acacia eriolobaSchinziophyton rautanenii association of the valley bottoms.

\section{Pterocarpus angolensis- Guibourtia coleosperma associa- tion of the Kavango Woodlands}

This association is characterized by the diagnostic species Pterocarpus angolensis, Cyperus margaritaceus, Eragrostis pallens, Burkea africana, Evolvulus alsinoides, Ochna pulchra, Tephrosia lupinifolia, Combretum psidioides, Stylosanthes fruticosa, Xenostegia tridentata subsp. angustifolia, Indigofera filipes, Polygala schinziana, Baphia massaiensis subsp. obovata var. obovata, Aristida stipitata, Tephrosia purpurea subsp. leptostachya var. pubescens, Rhynchosia venulosa, Baikiaea plurijuga, Securidaca longepedunculata, Ozoroa okavangensis, Bauhinia petersiana subsp. macrantha, Melinis repens subsp. grandiflora, Strychnos cocculoides, Megaloprotachne albescens, Combretum zeyheri, Stipagrostis uniplumis var. uniplumis, Lannea gossweileri, Heteropogon melanocarpus, Gardenia brachythamnus, Fadogia thamnus, Vernonia poskeana, Raphionacme lanceolata, Combretum collinum, Guibourtia coleosperma, Chamaecrista biensis, Commiphora tenuipetiolata, Aristida meridionalis, Diospyros chamaethamnus and Cyphostemma sandersonii. These are constantly associated by Terminalia sericea and Digitaria seriata. Twelve relevés have been classified into this association. A total of 145 species have been observed in this association, with on average 43.9 species per $1000 \mathrm{~m}^{2}$

This association represents the typical Kavango woodlands on the upper sand plateau (Fig. 6n), which have previously been described by Strohbach and Petersen (2007). These authors recognized three variants to the association, which could not be distinctly recognized during this study, as the sample set was too small. Likewise, mention is made by various authors of Baikiaea plurijuga-dominated woodlands (Burke 2002, De Sousa Correia \& Bredenkamp 1986, Mendelsohn \& el Obeid 2003). These could also not be clearly distinguished from the present data set. There is thus a clear need for further phytosociological investigation also in the relatively homogenous hinter- 
land to be able to resolve these conflicting observations.

\section{DISCUSSION}

\section{Higher order classification}

From the crispness calculations (BottaDukát et al. 2005) as depicted in Figure 3, a strong higher order classification is represented by the top four divisions. From the classification dendrogram in Figure 3 , the following higher orders can be identified:

- Wetlands, comprising associations $1.1,1.2$ and 1.3

- Saline grasslands, comprising association 2

- Floodplain \& valley bottom vegetation, comprising associations 3 , 4, 5, 6 and 7

- Sand vegetation, comprising associations 8 and 9 .

Again, due to the preliminary nature of this classification, no formal classification of these higher order syntaxa is offered.

Kangombe (2010) recognised four alliances in her classification of the vegetation of the Omusati and Oshana regions. One alliance, the Nymphaea nouchaliLeptochloa fusca alliance, represents the wetlands. This syntaxon can easily be represented by the three wetland associations distinguished in the present study.

A second alliance recognized by Kangombe (2010) is the Hyphaene petersiana-Acacia arenaria alliance. She includes here both various associations of saline grasslands as well as a Hyphaene petersiana dominated association. In the present study, the two similar associations (the Sporobolus ioclados- Willkommia sarmentosa association (2) and the Acacia tortilis-Hyphaene petersiana association (5) have been separated into separate higher syntaxa.

The third higher syntaxon identified in this study could be broadly described as part of the Acacietea as described by Volk \& Leippert (1971), as all contain at least some Acacia species. However, none represent typical examples of an Acaciadominated savanna similar to the Thornbush savanna sensu Giess (1998).

Strohbach and Petersen (2007) suggest that the Kavango woodlands vegetation is captured in a class Burkeo-Pterocarpetea. They include the shrub- and bushland vegetation dominated by Terminalia seriea, Combretum spp. and often Acacia fleckii also into this class. Also for the greater Omaheke (sand desert) of centraleastern Namibia, the vegetation could be classed into "hardeveld" and "sandveld" the latter including the broad-leafed savannas dominated by Terminalia sericea and Combretum spp, also including the Pterocarpus angolensis dominated vegetation. Yet, for this study, a suggested split of the true woodlands (dominated by trees like Pterocarpus angolensis) from the shrub- and bushlands dominated by the omnipresent Terminalia sericea and Combretum spp. into separate classes is suggested (Strohbach submitted, 2013).

The exact status of the higher-order syntaxa is thus unclear, and needs to be addressed in a synoptic revision of various phytosociological classifications within the broader Kalahari ecoregion.

\section{The vegetation associations within the Okavango River Valley land- scape}

Two factors influence the catena of vegetation associations within the Okavango valley in Kavango West: the depth of flooding and the soil type as determined through prehistoric and present flood regimes and aeolian sand deposition.

The depth and duration of flooding dictates how well the wetland associations develop - water depth, and especially in the case of the river bank, the steepness of the bank, dictate the composition and extend of the vegetation. It is also likely that vegetation, especially the reedbeds, act as landscape engineers, causing water to flow more slowly, allowing more sedimentation and subsequent re-channelling of the river bed (Gurnell 2013). Such processes have been reported as significant ecosystem engineers further downstream in the Okavango panhandle and Okavango Delta area (Ellery et al. 2003, Gumbricht et al. 2004, Tooth \& McCarthy 2004). Not only the wetland associations are directly dependent on the flooding depth, but also the general floodplain vegetation is dictated by present flood levels and past flooding and sedimentation patterns. This is illustrated in Figure 9.

The vegetation between the valley bottom and the top of the sand plateau is also distinctly dependent on the depth of sand. It is quite common to find psammophilous species like Guibourtia coleosperma within the valley bottom ecosystem - a

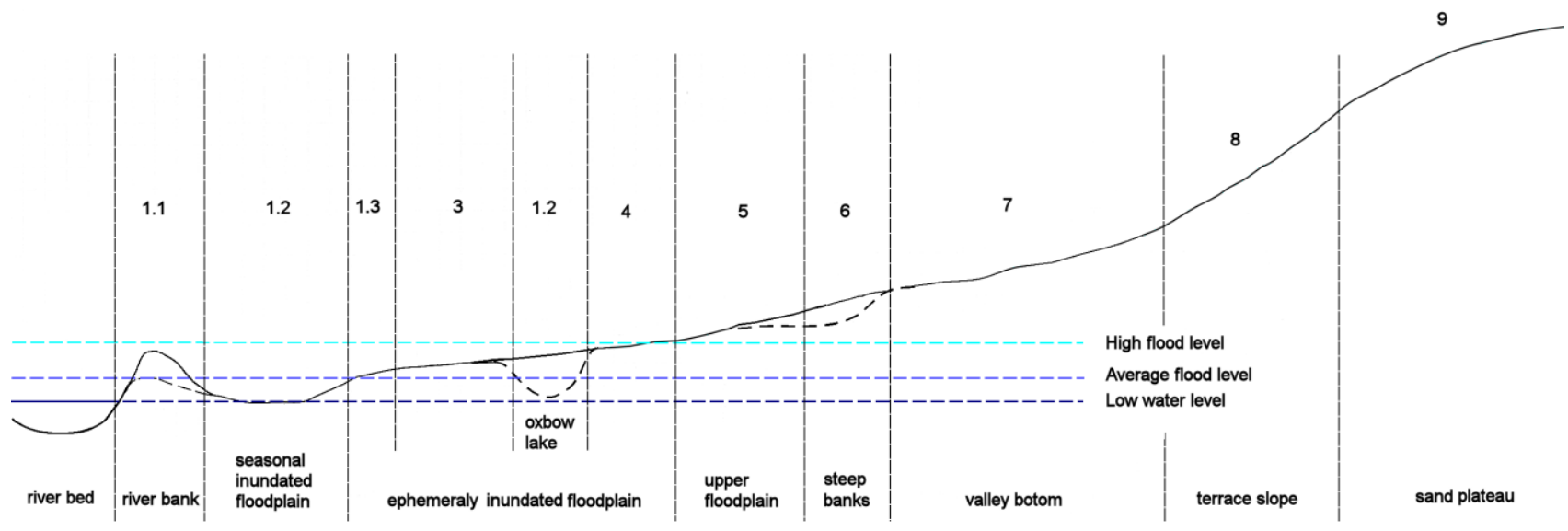

Fig. 9: Schematic sketch of the landscapes of the Okavango River valley. This sketch is neither to scale nor in correct proportions. The numbers indicate the approximate position of the associations in the landscape. Stippled lines represent alternative landscape forms which do not occur commonly. The Hakusembe grasslands (association 2) replace associations 3 and 4 in the landscape. 


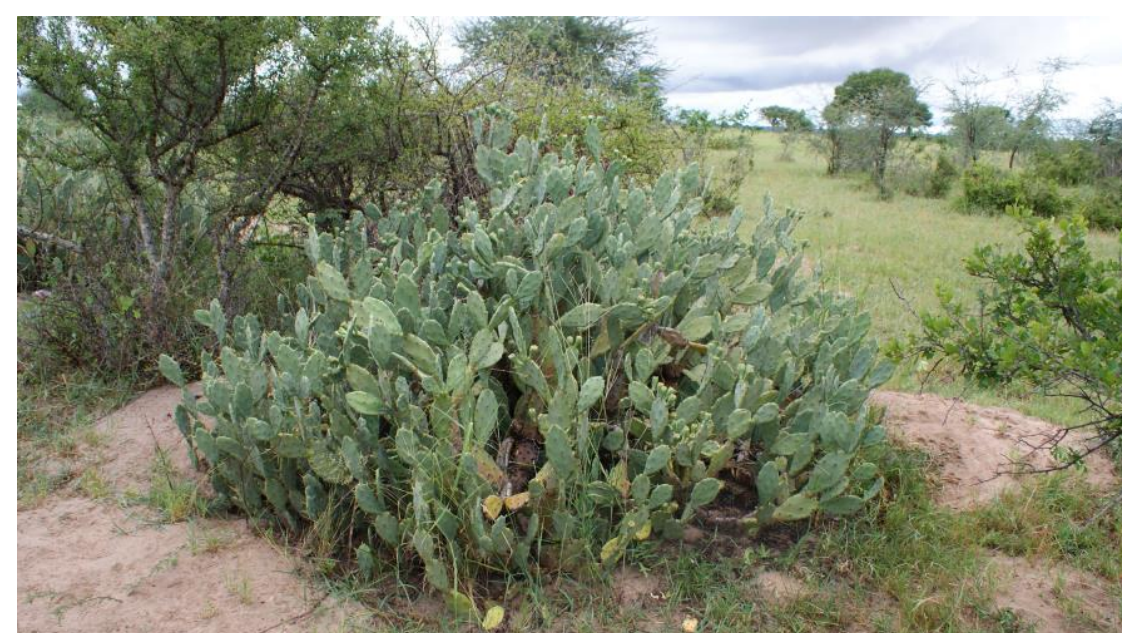

Figure 10: The alien invasive Opuntia stricta on the degraded Acacia eriolobaSchinziophyton rautanenii valley bottom.

sure sign that sand has been deposited within the valley in prehistoric times. As the valley bottom is however highly degraded, it is very difficult to tease apart such variations within the Acacia erioloba-Schinziophyton rautanenii association (7).

\section{Land use and conservation status}

As indicated before, the valley bottom is extensively utilized for arable agriculture, with well over $90 \%$ of the area having been ploughed (Hofmeyr 2004). Only limited patches of semi-natural vegetation of the Acacia erioloba-Schinzyophyton rautanenii association (7) still remain here. The relatively low number of species per relevé, and little variation in these numbers (Fig. 8) are indicative for the diversity-depleted state of this vegetation.

This pattern partially extends into both the Acacia tortilis-Hypahene petersiana association (5) (if with suitable soils) and the Acacia erioloba-Combretum collinum association (8). Observations are, however, that field expansions towards the terrace slopes are sporadic and driven by a shortage of land, not by their suitability for crops. Often such fields are also left semi-cleared, or are soon abandoned. The exact reason is unclear, but it is speculated that growing crops in the sandy soils does not produce a large yield, and that yields deteriorate with time, leading to the abandonment of these fields. This assumption is supported by the fact that the Kalahari sands are known to contain little nutrients (Pröpper et al. 2010, Thomas et al. 2000, Wang et al. 2007). Fears of further soil degradation, through a potential "slash and burn" type of agri-
Okavango river ecosystem (Aiyambo et al. in prep.).

As a general conclusion it can be said that the Okavango Valley vegetation is an immensely important ecosystem service provider, in which the ecosystem functioning and limits of resilience are not yet fully understood.

\section{Acknowledgments}

Many thanks to my colleagues Fransiska Kongombe and Salomé Kruger, who undertook the initial surveys and contributed with their data to the success of this undertaking. The National Herbarium of Namibia (WIND) kindly identified numerous unknown species we collected during our field surveys, especially those "difficult" aquatic species. The Hakusembe Lodge of the Gondwana Group provided a canoe for surveying on the floodplains free of charge. The soil samples were analysed by the Subdivision Analytical Services, Directorate Agricultural Research and Training, Ministry of Agriculture, Water and Forestry. The Division Hydrology of the same Ministry provided updated river flow level data for Rundu. The language editing, coupled with some valuable comments by Ms Shirley Bethune is gratefully acknowledged. This project was co-funded by the Ministry of Agriculture, Water and Forestry under their recurrent budget Vote 2004, as well as the German Federal Ministry of Education and Research under their FONA/Sustainable Land Management programme, under which the "Future Okavango" project is funded. severity of overgrazing on these floodplains is not clear. Again, extensive grazing within the seasonal wetlands, especially Oryza longistaminata or Oryzydium barnardii-dominated wetlands, but also excessive harvesting of reeds in the reedbeds, will lead to a degradation of fish spawning and fish breeding habitats. No clear signs of wood harvesting or deforestation were observed on the floodplains.

Another threat to the ecosystem of especially the upper floodplains and valley bottom vegetation is the immense encroaching potential of noxious alien invasive species. Very prominent on the Hakusembe floodplains are Opuntia stricta, which is partially planted as live hedges, but generally escaping from these controlled surroundings (Fig. 10). A total of 28 alien invasive species, ranging from annual herbaceous to perennial woody species, have been identified within the

\section{References}

Aiyambo, D. S., Klaassen, E. S., \& Strohbach, B. J. (in prep.): A preliminary survey of alien plants of the Kavango West and Kavango East Regions, Namibia. - Agricola.

Bethune, S. (1991): Kavango River Wetlands. - Madoqua 17(2): 77-112.

Biggs, R. C. (1976): The effects of the seasonal flood regime on the ecology of Chief's Island and the adjacent floodplain systems. In: The Okavango Delta and its Future Utilisation: 113-123. Gaborone.

Bonyongo, M. C. (1999): Vegetation ecology of the seasonal floodplains in the Okavango Delta, Botswana. - M.Sc. thesis, University of Pretoria. the agroclimate of Namibia. - M.Tech
Botha, L. (1996): Rainfall as an indicator of 
thesis, Faculty of Engineering, Technikon Pretoria.

Botta-Dukát, Z., Chytrý, M., Hájková, P., \& Havlová, M. (2005): Vegetation of lowland wet meadows along a climatic continentality gradient in Central Europe. Preslia 77: 89-111.

Brown, L. R., du Preez, P. J., Bezuidenhout, H., Bredenkamp, G. J., Mostert, T. H., Collins, N. B., \& Park, K. (2013): Guidelines for phytosociological classifications and descriptions of vegetation in southern Africa. - Koedoe 55: doi: 10.4102/koedoe.v55i1.1103.

Bruelheide, H. (2000): A new measure of fidelity and its application to defining species groups. - Journal of Vegetation Science 11: 167-178. CrossRef

Burke, A. (2002): Present Vegetation of the Kavango. - Journal of the Namibia Scientific Society 50: 133-145.

Central Bureau of Statistics. (2011): An Atlas of Poverty in Namibia. Windhoek, Namibia. - Windhoek: Central Bureau of Statistics.

Clarke, N. V. (1999): Flora of the Cuvelai wetlands, northern Namibia. Cimbebasia 15: 99-115.

Cunningham, A. B., Sullivan, S., \& Konstant, T. (1992): Palm utilisation and basketry resources in Owambo, northern Namibia. - Unpublished report.

De Sousa Correia, R. J., \& Bredenkamp, G. J. (1986): A reconnaissance survey of the vegetation of the Kavango, South West Africa. - Journal der SWA Wissenschaftlichen Gesellschaft XL/XLI: 29-45.

Ellery, K., Ellery, W. N., Rogers, K. H., \& Walker, B. H. (1991): Water depth and biotic insulation: Major determinants of back-swamp plant community composition. - Wetlands Ecology and Management 1(3): 149-162. CrossRef

Ellery, W., McCarthy, T., \& Smith, N. (2003): Vegetation, hydrology, and sedimentation patterns on the major distributary system of the Okavango fan, Botswana. - Wetlands 23(2): 357-375. CrossRef

Furness, H. D., \& Breen, C. M. (1980): The Vegetation of seasonally flooded areas of the Pongolo River Floodplain. - Bothalia 13(1\&2): 217-231.

Gauch, H. G. (1982): Multivariate analysis in community ecology. Cambridge: Cambridge University Press. CrossRef

Giess, W. (1998): A Preliminary Vegetation Map of Namibia. - Dinteria 4: 1-112.

Gumbricht, T., McCarthy, J., \& McCarthy, T. S. (2004): Channels, wetlands and islands in the Okavango Delta, Botswana, and their relation to hydrological and sedimentological processes. - Earth Surface Processes and Landforms 29(1): 15-29. CrossRef

Gurnell, A. (2013): Plants as river system engineers. - Earth Surface Processes and Landforms. $\underline{\text { CrossRef }}$
Hay, C. J., Van Zyl, B. J., \& Steyn, G. J. (1996): A quantitative assessment of the biotic integrity of the Okavango River, Namibia, based on fish. - Water SA 22: 263-284.

Hennekens, S. M., \& Schaminée, J. H. J. (2001): TURBOVEG, a comprehensive data base management system for vegetation data. - Journal of Vegetation Science 12(4): 589-591. CrossRef

Hill, M. O. (1973): Reciprocal Averaging: an Eigenvector method of ordination. Journal of Ecology 61(1): 237-249. CrossRef

Hill, M. O., \& Gauch, H. G. (1980): Detrended correspondence analysis: an improved ordination technique. - Vegetatio 42: 47-58. CrossRef

Hines, C. J. H. (1985): The birds of eastern Kavango, SWA/Namibia. - Journal of the South West African Scientific Society 40/41: 115-147.

Hines, C. J. H. (1993): Temporary wetlands of Bushmanland and Kavango; northeast Namibia. - Madoqua 18(2): 57-69.

Høberg, P., Lindholm, M., Ramberg, L., \& Hessen, D. O. (2002): Aquatic food web dynamics on a floodplain in the Okavango Delta, Botswana. - Hydrobiologia 470(1-3): 23-30. CrossRef

Hofmeyr, W. (Ed.). (2004): Proceedings of the Important Plant Areas Workshop. Windhoek: National Botanical Research Institute.

Junk, W. J., Bayley, P. B., \& Sparks, R. E. (1989): The flood pulse concept in riverfloodplain systems. - Canadian special publication of fisheries and aquatic sciences 106(1): 110-127.

Kangombe, F. N. (2010): The vegetation of Omusati and Oshana regions, centralnorthern Namibia. - M.Sc. thesis, University of Pretoria.

King, A. J., Humphries, P., \& Lake, P. S. (2003): Fish recruitment on floodplains: the roles of patterns of flooding and life history characteristics. - Canadian Journal of Fisheries and Aquatic Sciences 60(7): 773-786. CrossRef

Köppen, W. (1936): Das Geographische System der Klimate. (W. Köppen \& R. Geiger, [Eds.], Vol. 1 Part C. Berlin: Bornträger Verlag.

McCune, B., Grace, J. B., \& Urban, D. L. (2002): Analysis of Ecological Communities. - Gleneden Beach, Oregon: MjM Software.

Mendelsohn, J., \& el Obeid, S. (2003): Sand and Water. A profile of the Kavango Region. - Cape Town \& Windhoek: Struik Publishers \& RAISON.

Mendelsohn, J., \& el Obeid, S. (2004): Okavango River. The flow of a lifeline. Cape Town \& Windhoek: Struik Publishers \& RAISON.

Mendelsohn, J., Jarvis, A., Roberts, C., \& Robertson, T. (2002): Atlas of Namibia. - Cape Town: David Phillips Publishers.
Mendelsohn, J. M., el Obeid, S., De Klerk N., \& Vigne, P. (2006): Farming Systems in Namibia. - Windhoek: RAISON and Namibia National Farmers Union.

Merron, G. S., \& Bruton, M. N. (1995): Community ecology and conservation of the fishes of the Okavango Delta, Botswana. - Environmental Biology of Fishes 43(2): 109-119. CrossRef

Namibia Meteorological Services. (1997): Monthly and Annual Rainfall Normals for selected stations in Namibia which are used in the 10-Day Rainfall Bulletins. Windhoek: Namibia Meteorological Services.

National Planning Commission. (2012): Namibia 2011 Population and Housing Census Preliminary Results. - Windhoek: National Planning Commission.

Peck, J. E. (2010): Multivariate Analysis for Community Ecologists: Step-by-Step using PC-ORD. - Gleneden Beach, Oregon: MjM Software Design.

Pröpper, M., Gröngröft, A., Falk, T., Eschenbach, A., Fox, T., Gessner, U., Wisch, U. (2010): Causes and perspectives of land-cover change through expanding cultivation in Kavango. In: M. T. Hoffman, U. Schmiedel, N. Jürgens [Eds.]: - Implications for landuse and management. Vols. 1-3, Vol. 3, : 2-30. Göttingen \& Windhoek: Klaus Hess Publishers.

Roleček, J., Tichý, L., Zelený, D., \& Chytrý, M. (2009): Modified TWINSPAN classification in which the hierarchy respects cluster heterogeneity. - Journal of Vegetation Science 20: 596-602. CrossRef

Rössing Foundation. (2002): General palm planting and monitoring report from Kavango Region. - Windhoek: Rössing Foundation.

Schlesinger, W. H., Reynolds, J. F., Cunningham, G. L., Huenneke, L. F., Jarell, W. M., Virginia, R. A., \& Whitford, W. G. (1990): Biological feedbacks in Global Desertification. - Science 247: 10431048. CrossRef

Schlesinger, William H., \& Peterjohn, W. T. (1991): Processes controlling ammonia volatilization from Chihuahuan Desert soils. - Soil Biological Biochemistry 23(7): 637-642. CrossRef

Schneider, M. B. (1986): Notes on Terrace Soils of the Kavango River, northern SWA/Namibia. - Journal der SWA Wissenschaftlichen Gesellschaft XL/XLI: 199-213.

Strohbach, B., Cole, D., \& Seely, M. K. (2002): Environmental Impact Assessment on commercialisation of the basket industry depending on Makalani palm leaves as a resource in north-central Namibia. - Windhoek: Desert Research Foundation of Namibia.

Strohbach, B. J. (2001): Vegetation Survey of Namibia. - Journal of the Namibia Scientific Society 49: 93-124.

Strohbach, B., \& Kangombe, F. (2012): National Phytosociological Database of 
Namibia. - Biodiversity \& Ecology 4: 298. CrossRef

Strohbach, Ben J. (submitted): Vegetation of the Eastern Communal Conservancies in Namibia: I. Phytosociological descriptions. - Koedoe.

Strohbach, Ben J. (2012): Providing relevant, useful information on Namibian Vegetation Types. - Agricola 22: 7-39.

Strohbach, Ben J. (2013): Vegetation of the Eastern Communal Conservancies in Namibia: III. Annotated checklist. Dinteria 33: 3-42.

Strohbach, Ben J., \& Petersen, A. (2007): Vegetation of the central Kavango woodlands in Namibia: An example from the Mile 46 Livestock Development Centre. - South African Journal of Botany 37: 391-401. CrossRef

Sullivan, S., Konstant, T. L., \& Cunningham, A. B. (1995): The impact of utilization of palm products on the population structure of the Vegetable Ivory Palm (Hyphaene petersiana, Arecaceae) in North-Central Namibia. - Economic Botany 49(4): 357-370. CrossRef

Thomas, D. S. G., O'Connor, P. W., Bateman, M. D., Shaw, P. A., Stokes, S., \& Nash, D. J. (2000): Dune activity as a record of late Quaternary aridity in the Northern Kalahari: new evidence from northern Namibia interpreted in the context of regional arida and humid chronologies. - Palaeogeography, Palaeoclimatology, Palaeoecology 156: 243259. CrossRef

Thomas, David S. G., Knight, M., \& Wiggs, G. F. S. (2005): Remobilization of southern African desert dune systems by twenty-first century global warming. Nature 435(7046): 1218-1221. Cross$\underline{\text { Ref }}$

Tichý, L. (2002): JUICE, software for vegetation classification. - Journal of Vegetation Science 13: 451-453. CrossRef

Tooth, S., \& McCarthy, T. S. (2004): Controls on the transition from meandering to straight channels in the wetlands of the Okavango Delta, Botswana. - Earth Surface Processes and Landforms 29(13): 1627-1649. CrossRef

Vetter, S. (2001): Zambezian Baikiaea woodlands (AT0726). World Wildlife Fund. URL:

http://www.worldwildlife.org/wildworld/ profiles/terrestrial/at/at0726_full.html [assessed 28 July 2010].
Volk, O. H., \& Leippert, H. (1971): Vegetationsverhältnisse im Windhoeker Bergland, Südwestafrika. - Journal der SWA Wissenschaftliche Gesellschaft XXV: 544.

Wang, L., D'Odorico, P., Ringrose, S., Coetzee, S., \& Macko, S. A. (2007): Biogeochemistry of Kalahari sands. Journal of Arid Environments 71(3): 259-272. CrossRef

Weber, H. E., Moravec, J., \& Theurillat, J. P. (2000): International Code of Phytosociological Nomenclature. 3rd edition. Journal of Vegetation Science 11: 739768. $\underline{\text { CrossRef }}$

\section{Affiliation}

Ben J. Strohbach*

(bstrohbach@polytechnic.edu.na)

School of Natural Resources and Spatial

Sciences, Polytechnic of Namibia

P/Bag 13388

Windhoek, NAMIBIA

*Corresponding author 
